\title{
Tissue homeostasis and adaptation to immune challenge resolved by fibroblast network mechanics
}

Harry L. Horsnell ${ }^{1}$, Robert J. Tetley ${ }^{2}$, Henry De Belly ${ }^{3}$, Spyridon Makris ${ }^{1}$, Lindsey J. Millward ${ }^{1}$, Agnesska C. Benjamin ${ }^{1}$, Charlotte M de Winde ${ }^{1}$, Ewa K. Paluch ${ }^{3}$, Yanlan $\mathrm{Mao}^{2,4}$, Sophie E. Acton ${ }^{1 \#}$

1. Stromal Immunology Group, MRC Laboratory for Molecular Cell Biology, University College London, Gower Street, London, WC1E 6BT, United Kingdom

2. Tissue Mechanics Group, MRC Laboratory for Molecular Cell Biology, University College London, Gower Street, London, WC1E 6BT, United Kingdom

3. Physiological Laboratory, Department of Physiology, Development and Neuroscience, University of Cambridge, CB2 3EG, United Kingdom

4. Institute for the Physics of Living Systems, University College London, Gower Street, London WC1E 6BT, UK

\# Corresponding author Sophie E. Acton: $\underline{\text { s.acton@ucl.ac.uk }}$

\section{Key words}

Lymph node

Fibroblastic reticular cell

Podoplanin

Network tension

Tissue remodelling 


\section{Abstract}

3 Emergent physical properties of tissues are not readily understood by reductionist studies of their 4 constituent cells. Here, we show molecular signals controlling cellular physical properties, 5 collectively determining tissue mechanics of lymph nodes, an immunologically-relevant, adult 6 mammalian tissue. Lymph nodes paradoxically maintain robust tissue architecture in homeostasis 7 yet are continually poised for extensive tissue expansion upon immune challenge. We find that 8 following immune challenge, cytoskeletal mechanics of a cellular meshwork of fibroblasts determine 9 tissue tension independently of extracellular matrix scaffolds. We determine that CLEC102 /podoplanin signalling regulates the cell surface mechanics of fibroblasts, permitting cell elongation 11 and interdigitation through expedited access to plasma membrane reservoirs. Increased tissue 12 tension through the stromal meshwork gates the initiation of fibroblast proliferation, restoring 13 homeostatic cellular ratios and tissue structure through expansion. 
14 Unlike developmental systems that display progressive tissue growth and maturation ${ }^{1}$, the 15 homeostatic state of adult tissues is robust, maintaining form and function ${ }^{2}$. Secondary lymphoid 16 organs, are uniquely able to dramatically change size in response to immune challenge, adapting to 17 the increased space requirements of millions of infiltrating and proliferating lymphocytes, while 18 remaining structurally and functionally intact throughout ${ }^{3-5}$. As a mechanical system, lymph nodes 19 continually resist and buffer the forces exerted by trafficking of lymphocytes entering and leaving 20 the tissue in steady state $^{6}$, and managing diurnal fluctuations in cell trafficking ${ }^{7}$. Tissue size is 21 determined by lymphocyte numbers, highlighted by the small organ size in genetic models blocking 22 lymphocyte development $(\operatorname{Rag} 1 \mathrm{KO})^{8}$, and the ability of the tissue to expand 2-10 fold in size to 23 accommodate lymphocyte proliferation through adaptive immune responses ${ }^{3-5,9}$.

24 Lymph nodes function at the interface of immunity and fluid homeostasis, constructing a physical 325 dimensional cellular meshwork linking fluid flow, immune surveillance and adaptive immunity ${ }^{10}$. The most populous stromal cell component are fibroblastic reticular cells (FRCs) which span the whole tissue generating a interconnected cellular network with small world properties ${ }^{11}$, forming robust clustered nodes with short path lengths, and surrounding bundles of extracellular matrix fibres ${ }^{12}$. It is widely assumed that extracellular matrix scaffolds are the predominant force-bearing structures determining tissue mechanics ${ }^{1,2}$. However the relative contributions of the cellular structures versus the underlying extracellular network to tissue mechanics have not been addressed in a highly cellularised system undergoing such extensive expansion ${ }^{2}$. As tissue size and cellularity increase in response to immunogenic challenge, it is known that lymph nodes become more deformable when challenged with compressive force ${ }^{3}$. During the remodelling process the FRC network maintains connectivity through the elongation and increased spacing between FRCs, increasing mesh size of the network ${ }^{3}$. It is also known that $\mathrm{CLEC}-2^{+}$dendritic cells are required to prime the stromal architecture for tissue expansion ${ }^{3,4}$ but the downstream impacts on the mechanical properties of the cellular network and extracellular matrix scaffolds driving the adaptation in tissue mechanics are unknown. During tissue expansion, FRCs reduce their adhesion to the underlying extracellular matrix bundles and these matrix scaffolds become fragmented ${ }^{9}$. This makes lymph nodes an ideal model system to address the relative mechanical contributions of cellular and material structures to emergent tissue mechanics.

43 As fibroblasts are contractile, force-generating cells ${ }^{13}$, we hypothesise that the interconnected FRC 44 network determines tissue mechanics. The FRC network, identified by podoplanin expression, spans 45 the whole lymph node tissue but specifically supports $\mathrm{CD}^{+} \mathrm{T}$ cell function in the paracortex, 46 providing trafficking routes from high endothelial venules to $B$ cell follicles ${ }^{14,15,16,17}$ (Fig. 1A-B). We 
47 asked how the FRC network and associated extracellular matrix scaffolds participate in tissue 48 mechanics and architecture in the immunological steady state. Following laser ablation (Fig. 1C-D, 49 and supplementary Movie. S1A), we measured a mean recoil of $0.42 \mu \mathrm{m} / \mathrm{s}^{-1}$ in the fibroblastic 50 reticular network of the paracortex, using a fibroblast-specific membrane-eGFP mouse model 51 (PDGFR $\alpha$-mGFP-CreERT2 (Fig. 1C, Fig. S1, Fig. S2A), formally demonstrating that the reticular 52 network is under mechanical tension in the tissue (Fig. 1E).

53 To investigate how cellular-scale components of the stromal architecture contribute to tissue 54 mechanics, we examined the cytoskeletal and extracellular matrix structures of the reticular network. In steady state, FRCs tightly adhere to and enwrap collagen bundles forming a continuous meshwork $^{18}$. Therefore, recoil following laser ablation is a measure of the combined mechanical properties of both the cell and the extracellular matrix structures in steady state (Fig. 1C-F). Maximum z-projections and orthogonal views of FRCs show F-actin cables aligned proximal to the collagen bundles and spanning beneath the T-cell-facing FRC plasma membrane (Fig. 1F, and supplementary Movie. S1B). These F-actin structures co-localised with phosphorylated myosin regulatory light chain $(\mathrm{pMLC} 2)^{19}$ (Fig. 1F), indicating that FRCs are contractile and resisting strain in steady state ${ }^{20}$. Since resting lymph node size is constant, forces are balanced in the steady state tissue.

We next asked how the reticular network reacts to the forces exerted by increasing numbers of lymphocytes following immune challenge. It has been observed that the network of bundled matrix fibres becomes fragmented ${ }^{9}$, while the cellular component of the meshwork remains intact and connected $^{3,4,11}$. Therefore, we next tested if the intact cellular network could compensate for the loss of extracellular matrix integrity to balance tissue tension. In response to immunisation with incomplete Freund's adjuvant and ovalbumin (IFA/OVA), the tissue expands 2-3 fold over the first 5 days (Fig. 2A). Surprisingly, at day 3 post-immunisation, initial recoil velocity was decreased by $29 \%$ to $0.30 \mu \mathrm{m} / \mathrm{s}^{-1}$ (Fig. 2B-D, and Supplementary Movie. S2A) despite a 1.5-fold increase in tissue mass (Fig 2A). In contrast, 2 days later (at day 5 post-immunisation), mean initial recoil velocity was $60 \%$ higher than in the steady state at $0.71 \mu \mathrm{m} / \mathrm{s}^{-1}$ (Fig. 2B-D, and supplementary Movie. 2B). Since the extracellular matrix scaffold is fragmented at this phase of tissue expansion ${ }^{9}$, we hypothesised that the actin cytoskeleton must be the major contributor to the increased tissue tension. Indeed, recoil measurements following laser ablation of tissues pre-treated with ROCK inhibitor (Y27632) to inhibit cytoskeletal contractility ${ }^{19}$, demonstrated that tissue tension was reduced to basal levels at all time

78 points tested (Fig. 2C, Fig. S2B-F). These results indicate that tissue tension is determined by 79 differential resistive actomyosin forces (Fig.2C). 
805 days post immunisation, the whole tissue is more deformable ${ }^{3}$, yet tension measured through the 81 fibroblastic reticular network increases (Fig. 2B-C). Since elasticity of gels is known to scale as the 82 inverse of mesh size $e^{21}$, the combination of the fragmentation of the matrix and increasing mesh size 83 of the FRC network may explain increased tissue deformability through acute expansion. To 84 investigate how the FRC network responds mechanically to the increasing tissue size (Fig. 2A) we compared the cytoskeletal and matrix structures of the FRCs at day 3 (lower tension) versus day 5 (higher tension). In reactive lymph nodes, 3 days after IFA/OVA immunisation, F-actin structures were located primarily proximal to the basement membrane (asterisk), but T-cell facing F-actin cables were absent (Fig. 2E, and Supplementary Movie S2C). However, 5 days post immunisation, prominent contractile F-actin cables structures span the length of FRC cell bodies even in the absence of underlying matrix bundles (arrowhead) (Fig. 2E, and supplementary Movie S2D). These data show that tissue tension varies in response to immunogenic challenge and that increased tissue tension occurs independently of extracellular matrix integrity. Instead, mechanical forces are generated by increased packing of lymphocytes in the FRC meshwork and resisted by actomyosin through the FRC network (Fig. 2).

Since the FRC network is not resisting increased packing of lymphocytes at day 3 (Fig. 2C), regulation of actomyosin contractility alone cannot explain how the FRC network remains connected as the tissue mass increases (Fig. 2A). We next sought to understand the mechanisms controlling the cell surface mechanics of FRCs. To increase mesh size without increasing FRC number ${ }^{3,4}$; FRCs must elongate or make protrusions, adapting their cell morphology to maintain network integrity ${ }^{3,11}$.

100 Effective membrane tension (hereafter membrane tension), is determined by the in-plane tension of 101 the lipid bilayer and the strength of membrane-to-cortex attachments ${ }^{22,23}$ and can regulate cell 102 spreading, changes in morphology and cell fate $\mathrm{e}^{22-24}$. Cell contact between antigen-presenting 103 dendritic cells and FRCs through CLEC-2/podoplanin binding, peaking at day 3 , is known to regulate 104 lymph node deformability and tissue expansion ${ }^{3,4}$. Therefore, we asked whether the 105 CLEC2/podoplanin interaction regulates FRC cell surface mechanics. We used optical tweezers to 106 measure membrane tension of FRCS, (Fig. 3A, Fig. S3G, and supplementary Movie. S3A) and find that 107 specific engagement of CLEC-2 to podoplanin reduces membrane tension (Fig. 3A-B) and 108 downregulates phosphorylation of ezrin, radixin and moesin family proteins (pERM) (Fig. S3F), which 109 tether the actin cytoskeleton to the plasma membrane. Signalling specificity was confirmed by 110 exogenous expression of a PDPN mutant that cannot bind CLEC-2 (T34A) and a mutant which cannot 111 signal through the cytoplasmic tail (S167A-S171A) (Fig. S3A-D). Both showed no change in 112 membrane tension in the presence of CLEC-2 (Fig. S3A-D). As podoplanin interacts with CD44 ${ }^{25}$, also 113 known to bind ezrin ${ }^{26}$, we tested the relatively contributions of both CD44 and podoplanin to 
114 membrane tension in unstimulated FRCs and find that podoplanin is the key driver in steady state

115 (Fig. 3C, Fig. S3E)

116 In the tissue, FRCs must each elongate and form protrusions to interdigitate with their network 117 neighbours as they spread and expand the stromal cell network. These cell shape changes can be 118 achieved by increasing the ratio of plasma membrane surface area to cell volume through exocytotic 119 pathways or by unfolding membrane reservoirs ${ }^{27}$. FRCs contain active EHD2 ${ }^{+}$caveolae structures $^{28}$ 120 contributing to tension-sensitive plasma membrane reservoirs (Fig. 3D). We tested, using osmotic 121 shock (Fig. S4A-B) whether regulation of membrane tension through the CLEC-2/podoplanin 122 signalling axis impacted how rapidly FRC could utilise existing membrane reservoirs to respond to 123 the external forces (Fig 3E-F). We found that CLEC-2 engagement to podoplanin (Fig. 3E, Fig. S4C, 124 and supplementary Movie. S4-B) or knockdown of podoplanin (PDPN KD) (Fig. 3F, and 125 supplementary Movie. S4C) permitted more rapid cell expansion in hypotonic conditions but did not 126 alter total membrane availability (Fig. S4D, and supplementary Movie. S4D). In vivo, we observed 127 individual, differentially labelled, neighbouring FRCs increasing cell-cell contact 3 days post 128 immunisation (Fig. 3G), suggesting that additional plasma membrane is used for morphological 129 adaptations and incorporated into cell extensions. We propose that changing cell surface mechanics 130 of FRCs in combination with reduced actomyosin resistance (Fig. 2C) permits lymph node expansion 131 whilst maintaining FRC network integrity.

132 However, at day 5, even larger number of lymphocytes puts the network under increased tension 133 which is resisted by actomyosin contractility (Fig. 2C). We asked what the functional consequence of 134 changing tissue tension had on tissue remodelling in response to immune challenge. Existing studies 135 have found that expansion of FRC populations lag behind lymphocytes ${ }^{3-5}$, but it is not known how 136 FRC proliferation is triggered or spatially regulated within the tissue. 5 days post IFA/OVA 137 immunisation the number of proliferating FRCs (Ki67 ${ }^{+}$) doubles compared to steady state (Fig. 4A-B), 138 which correlates with increased tissue tension (Fig. 2B-D). Ki67 $7^{+}$proliferative FRCs were observed 139 throughout the tissue, and we observed no specific proliferative niche surrounding blood vessels or 140 beneath the bounding capsule (Fig. 4A), suggesting that the cue for entry into the cell cycle is not 141 spatially restricted or limited to a subpopulation of FRCs. We hypothesised that increased 142 mechanical tension may gate FRC entry into cell division. To test this, we blocked the increase in 143 tissue tension unilaterally in vivo through pharmacological inhibition of ROCK ${ }^{19}$ (Fig. 4C-D) and found 144 that proliferation of stromal cells, specifically the FRC population, was significantly attenuated 5 days 145 post immunisation, while lymphocyte proliferation and the increases in tissue mass and cellularity 146 were unaffected (Fig. 4E-I, Fig. S5A-D). This leads us to conclude that the stromal architecture is 
147 reactive to the physical space requirements of the lymphocyte populations. Indeed, previous studies

148 have identified a remarkably robust ratio between fibroblastic stroma and T cells, maintained as the 149 tissue expands ${ }^{29}$. A mechanical cue for stromal cell growth and proliferation would ensure that the 150 steady state ratio of fibroblastic stroma to lymphocytes is perfectly restored, independently of the 151 kinetics or scale of the immune reaction. Homeostatic tissue architecture is recovered as the tissue expands reinstating the supportive immune microenvironment for lymphocyte populations ${ }^{30}$.

153 Together our data demonstrate that the fibroblastic structure of the lymph node is the active 154 mechanical component during tissue expansion. Using the dynamic cellular network rather than the more rigid ECM to respond to changing lymphocytes numbers in the tissue provides the lymph node 156 with an elegant mechanical system that can proportionately respond to lymphocyte requirements. 157 We show that the lymph node becomes mechanically permissive to expansion through CLEC-2/PDPN 158 signalling priming the cell surface mechanics of FRCs. As the kinetics of expansion increase tissue 159 tension, FRC's initiate cellular division to restore tissue architecture. Other studies have shown that 160 tissue scale properties emerge from cellular scale mechanics in the transition from developing to 161 adult tissue ${ }^{31}$. We now directly address this concept in an immunological relevant adult mammalian 162 tissue during homeostasis and immune challenge. We show that the interconnected cellular network 163 deploys molecular signals controlling cellular mechanical properties to collectively determine tissue 164 scale mechanics of lymph nodes. 


\section{References}

1. Petridou, N. I., Spiró, Z. \& Heisenberg, C. P. Multiscale force sensing in development. Nature Cell Biology vol. 19 581-588 (2017).

2. Wyatt, T., Baum, B. \& Charras, G. A question of time: Tissue adaptation to mechanical forces. Current Opinion in Cell Biology vol. 38 68-73 (2016).

3. Acton, S. E. et al. Dendritic cells control fibroblastic reticular network tension and lymph node expansion. Nature 514, 498-502 (2014).

4. Astarita, J. L. et al. The CLEC-2-podoplanin axis controls the contractility of fibroblastic reticular cells and lymph node microarchitecture. Nat. Immunol. 16, 75-84 (2015).

5. Yang, C.-Y. et al. Trapping of naive lymphocytes triggers rapid growth and remodeling of the fibroblast network in reactive murine lymph nodes. Proc. Natl. Acad. Sci. 111, E109-E118 (2014).

6. Mandl, J. N. et al. Quantification of lymph node transit times reveals differences in antigen surveillance strategies of naïve CD4+ and CD8+ T cells. Proc. Natl. Acad. Sci. U. S. A. 109, 18036-18041 (2012).

7. Druzd, D. et al. Lymphocyte Circadian Clocks Control Lymph Node Trafficking and Adaptive Immune Responses. Immunity 46, 120-132 (2017).

8. Kim, J.-I. et al. CRISPR/Cas9-mediated knockout of Rag-2 causes systemic lymphopenia with hypoplastic lymphoid organs in FVB mice. Lab Anim Res 34, 166-175 (2018).

9. Martinez, V. G. et al. Fibroblastic Reticular Cells Control Con duit Matrix Deposition during Lymph Node Expansion Article Fibroblastic Reticular Cells Control Conduit Matrix Deposition during Lymph Node Expansion. CellReports 29, 2810-2822.e5 (2019).

10. Krishnamurty, A. T. \& Turley, S. J. Lymph node stromal cells: cartographers of the immune system. Nature Immunology vol. 21 369-380 (2020).

11. Novkovic, M. et al. Topological Small-World Organization of the Fibroblastic Reticular Cell Network Determines Lymph Node Functionality. PLoS Biol. 14, 1-20 (2016).

12. Malhotra, D. et al. Transcriptional profiling of stroma from inflamed and resting lymph nodes defines immunological hallmarks. Nat. Immunol. 13, 499-510 (2012).

13. Sahai, E. et al. A framework for advancing our understanding of cancer-associated fibroblasts. Nat. Rev. Cancer doi:10.1038/s41568-019-0238-1.

14. Bajénoff, M. et al. Stromal Cell Networks Regulate Lymphocyte Entry, Migration, and Territoriality in Lymph Nodes. Immunity 25, 989-1001 (2006).

15. Bajénoff, M., Glaichenhaus, N. \& Germain, R. N. Fibroblastic Reticular Cells Guide T Lymphocyte Entry into and Migration within the Splenic T Cell Zone. J. Immunol. 181, 39473954 (2008).

16. Herzog, B. H. et al. Podoplanin maintains high endothelial venule integrity by interacting with platelet CLEC-2. Nature 502, 105-109 (2013). 
17. Cremasco, V. et al. B cell homeostasis and follicle confines are governed by fibroblastic reticular cells. Nat. Immunol. 15, 973-981 (2014).

18. Gretz, J. E., Anderson, a O. \& Shaw, S. Cords, channels, corridors and conduits: critical architectural elements facilitating cell interactions in the lymph node cortex. Immunol. Rev. 156, 11-24 (1997).

19. Amano, M. et al. Phosphorylation and activation of myosin by Rho-associated kinase (Rhokinase). J. Biol. Chem. 271, 20246-20249 (1996).

20. Étienne, J. et al. Cells as liquid motors: Mechanosensitivity emerges from collective dynamics of actomyosin cortex. Proc. Natl. Acad. Sci. U. S. A. 112, 2740-2745 (2015).

21. Hoshino, K.-I., Nakajima, T., Matsuda, T., Sakai, T. \& Gong, J. P. Network elasticity of a model hydrogel as a function of swelling ratio: from shrinking to extreme swelling states + . Soft Matter 14, 9693 (2018).

22. Lieber, A. D., Yehudai-Resheff, S., Barnhart, E. L., Theriot, J. A. \& Keren, K. Membrane tension in rapidly moving cells is determined by cytoskeletal forces. Curr. Biol. 23, 1409-1417 (2013).

23. Diz-Muñoz, A. et al. Membrane Tension Acts Through PLD2 and mTORC2 to Limit Actin Network Assembly During Neutrophil Migration. PLOS Biol. 14, (2016).

24. De Belly, H. et al. Membrane Tension Gates ERK-Mediated Regulation of Pluripotent Cell Fate. Cell Stem Cell 28, 273-284.e6 (2021).

25. de Winde, C. M. et al. Fibroblastic reticular cell response to dendritic cells requires coordinated activity of podoplanin, CD44 and CD9. J. Cell Sci. (2021) doi:10.1242/JCS.258610.

26. Martín-Villar, E. et al. Podoplanin Associates with CD44 to Promote Directional Cell Migration. Mol. Biol. Cell 21, 4387-4399 (2010).

27. Salbreux, G., Charras, G. \& Paluch, E. Actin cortex mechanics and cellular morphogenesis. Trends Cell Biol. 22, 536-545 (2012).

28. Torrino, S. et al. EHD2 is a mechanotransducer connecting caveolae dynamics with gene transcription. Prep. (2018) doi:10.1083/JCB.201801122.

29. Yang, C.-Y. et al. Trapping of naive lymphocytes triggers rapid growth and remodeling of the fibroblast network in reactive murine lymph nodes. Proc. Natl. Acad. Sci. 111, E109-E118 (2014).

30. Link, A. et al. Fibroblastic reticular cells in lymph nodes regulate the homeostasis of naive T cells. Nat. Immunol. 8, 1255-1265 (2007).

31. McGinn, J. et al. A biomechanical switch regulates the transition towards homeostasis in oesophageal epithelium. Nat. Cell Biol. 23, 511-525 (2021). 


\section{Acknowledgements}

We thank Prof E. Sahai, Prof G. Charras and Dr V.G. Martinez for critical reading of the manuscript. We also thank Prof. H. Clevers for supplying R26R-confetti mice.

\section{Funding}

This work was supported by the European Research Council Starting Grant (LNEXPANDS; to S.E.A), Cancer Research UK Careers Development Fellowship (CRUK-A19763; to S.E.A), Medical Research Council (MC-U12266B), MRC awards MR/L009056/1 and MR/T031646/1 (Y.M), Lister Institute Research Prize and EMBO Young Investigator Programme (Y.M), the European Union's Horizon 2020 research and innovation programme under the Marie Sklodowska-Curie grant agreement No 641639 (ITN Biopol, E.K.P) and the Medical Research Council UK (MRC programme award MC_UU_12018/5 (E.K.P).

\section{Author contributions}

H.L.H and S.E.A designed the study and wrote the manuscript. H.L.H performed and analysed all experiments. A.C.B. and S.E.A. generated the PDGFR $\alpha$-mGFP-CreERT2 mouse model. R.J.T assisted with laser ablation studies. H.d.B assisted with optical trap measurements. H.d.B contribution was carried out under the support of E.K.P at MRC-LMCB UCL, current location is the department of Biochemistry and Biophysics, UCSF, San Francisco, CA, USA. S.M assisted with flow cytometry of stromal cells. L.J.M conducted imaging experiments of FRCS in vivo. C.M.d.W. generated CD44 KO cell lines. Y.M and E.K.P contributed to study design and editing of the manuscript. All authors contributed to editing of the manuscript.

\section{Competing interests}

The authors declare no competing interests

\section{Supplementary Materials}

Materials and methods

Figs. S1 to S5

Table S1

References (1-14)

Movies S1 to S4 
A

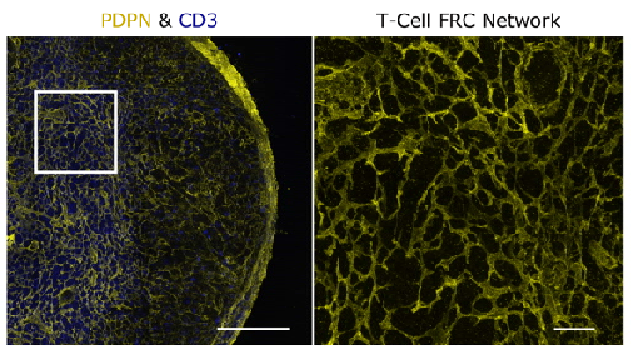

C

ROI (mGFP)

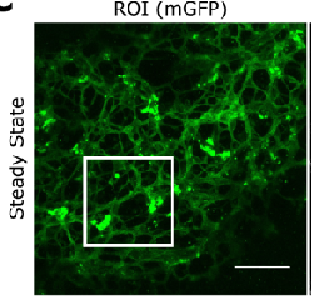

Ablation Site
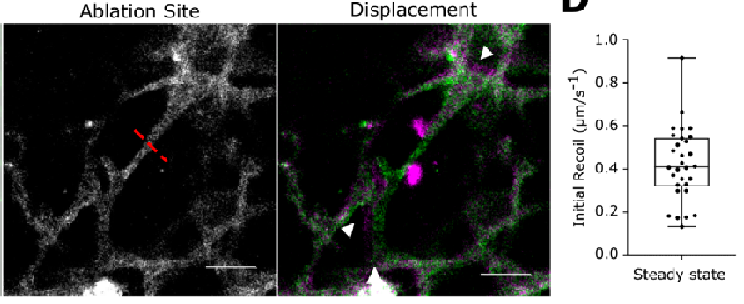

D

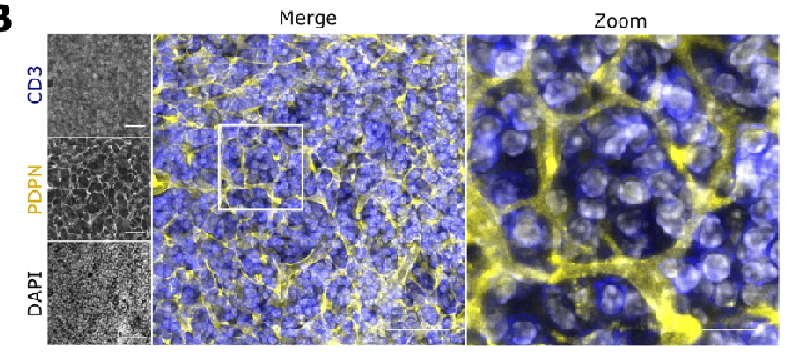

$\mathbf{F}$

Perlecan

PDPN

F-actin

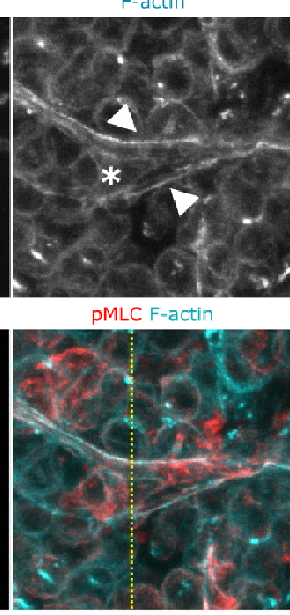

$\mathrm{pMLC}$

$\mathbf{E}$

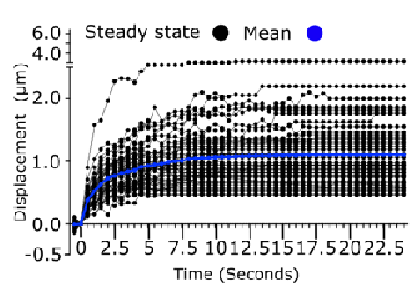

Time (Seconds)

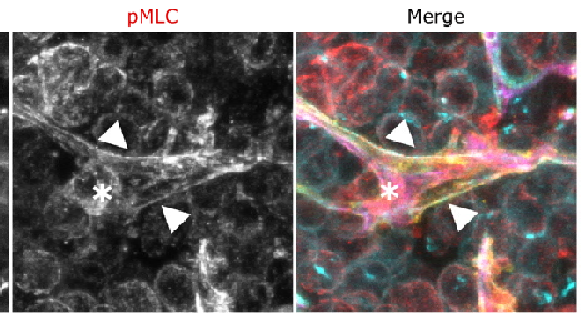

Orthogonal

Orthogonal
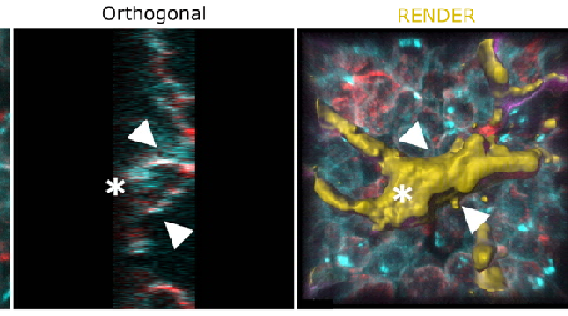

Fig. 1 to 4

Figure 1 - Lymph node tissue tension measured through the FRC network

A) Lymph node tile scan (left), paracortex (right) maximum Z-projection. PDPN (FRCs, yellow), CD3 (T-cells, blue). Scale bar $500 \mathrm{~m}$ (left), $25 \mathrm{~m}$ (right). B) FRC network structure and T-cell compaction vibratome slices. PDPN (FRCs), CD3 (T-cells), DAPI (Nuclei). Scale bar $\quad$ (left), $\quad$ (right). C) PDGFR -mGFP (FRCs) ablation ROI (white box) and cut site (red dotted line). Scale bar. Recoil displacement (white arrowheads) with pre (green) and post cut (magenta) overlay. Scale bar D) Initial recoil velocity $\left(\mathrm{m} / \mathrm{s}^{-1}\right)$. Box plot indicates median, interquartile range and $\min / \mathrm{max}$. E) Individual recoil curves (black) compared to the mean (blue). N=5 LNs. F) Paracortical steady state 
bioRxiv preprint doi: https://doi.org/10.1101/2021.05.27.446027; this version posted July 30, 2021. The copyright holder for this preprint (which was not certified by peer review) is the author/funder, who has granted bioRxiv a license to display the preprint in perpetuity. It is made available under aCC-BY-NC-ND 4.0 International license.

FRCs. Perlecan (matrix), PDPN (FRC), phalloidin (F-actin) and pMLC. Asterisk and arrowheads indicate F-actin cables. Orthogonal views (yellow dotted line, YZ axis, $10 \mu \mathrm{m}$ depth). Scale bar $10 \mu \mathrm{m}$. 

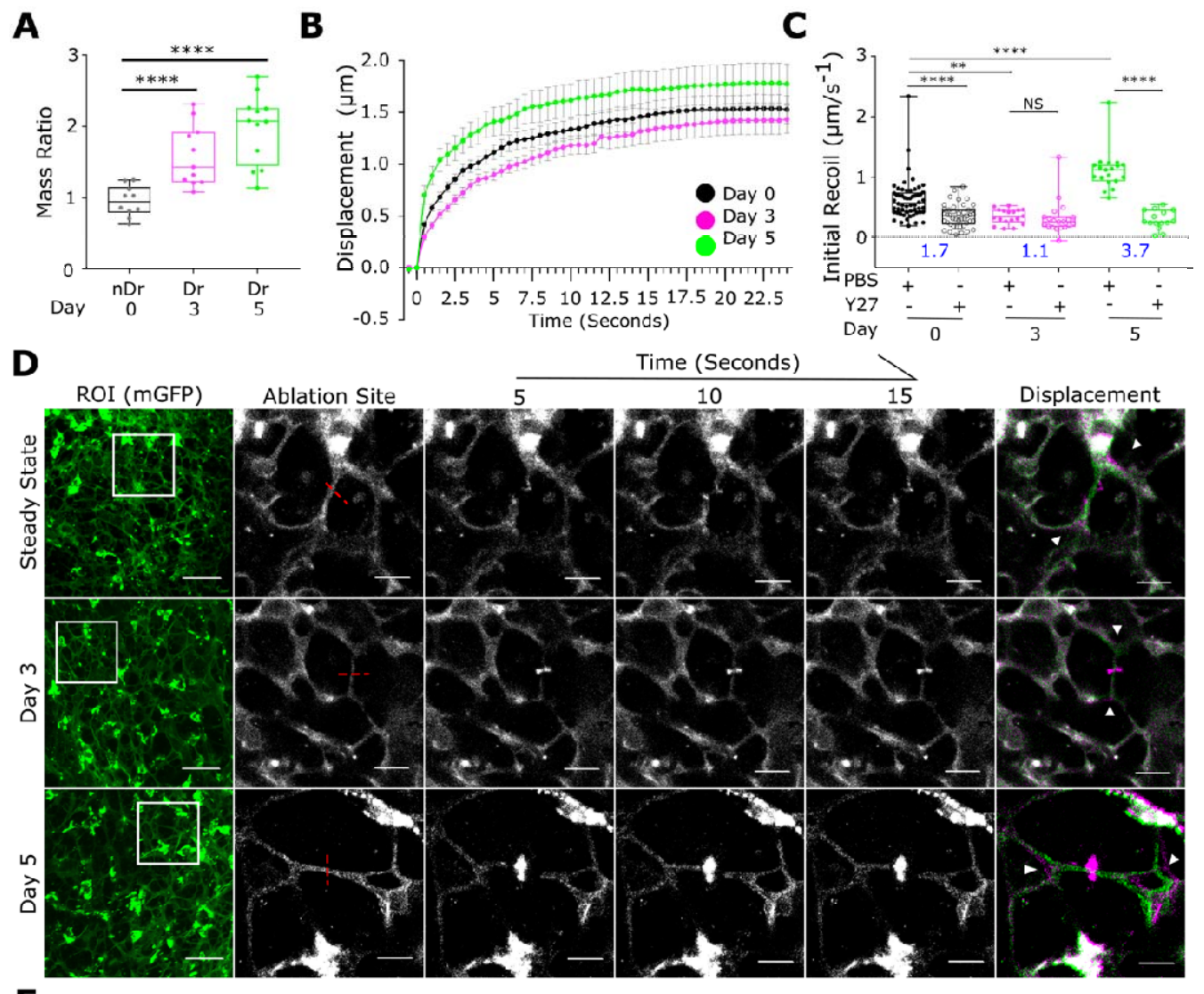

$\mathbf{E}$

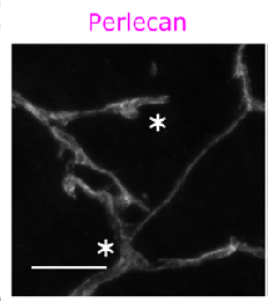

PDPN

F-actin

PMLC

Merge
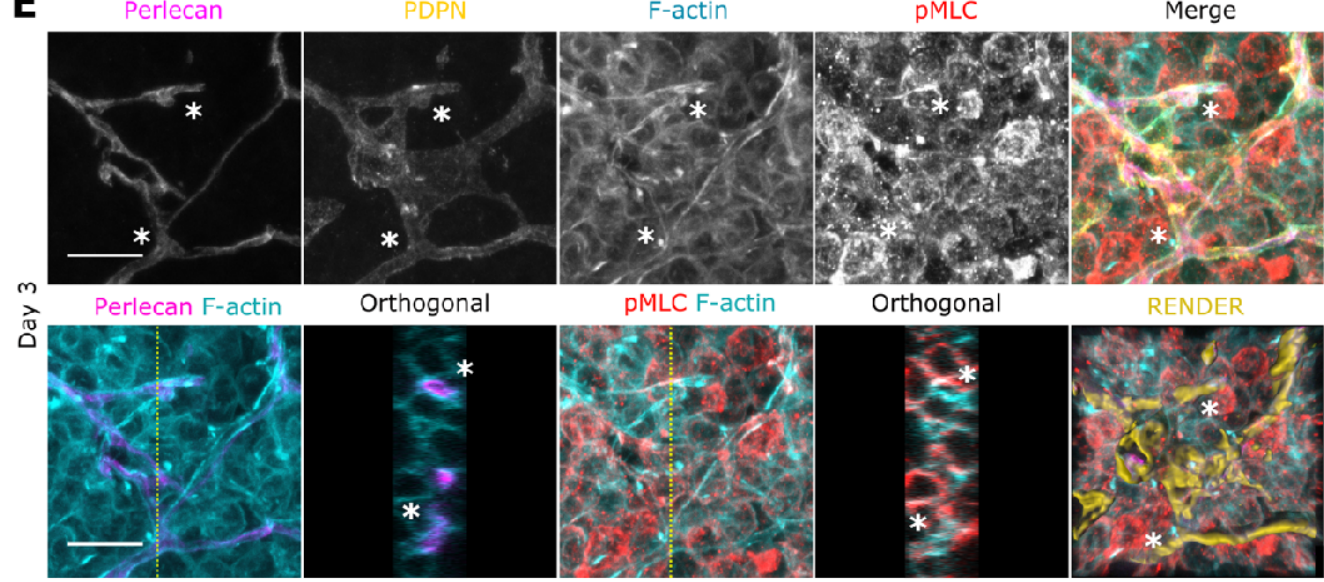

pMLC F-actin

Orthogonal
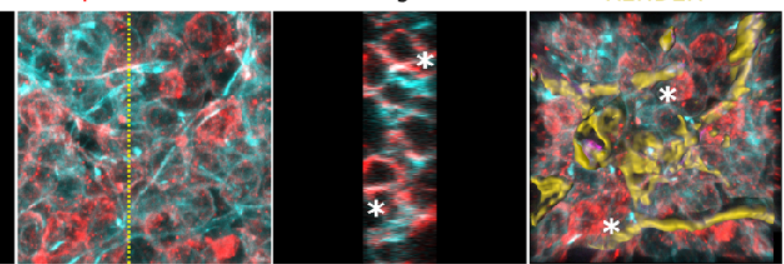

Perlecan

F-actin

pMLC

Merge
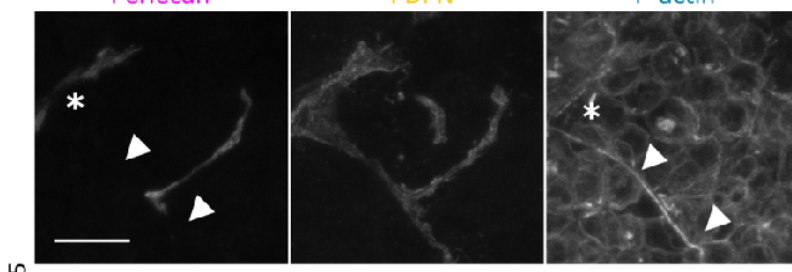

pMLC F-actin

Orthogonal
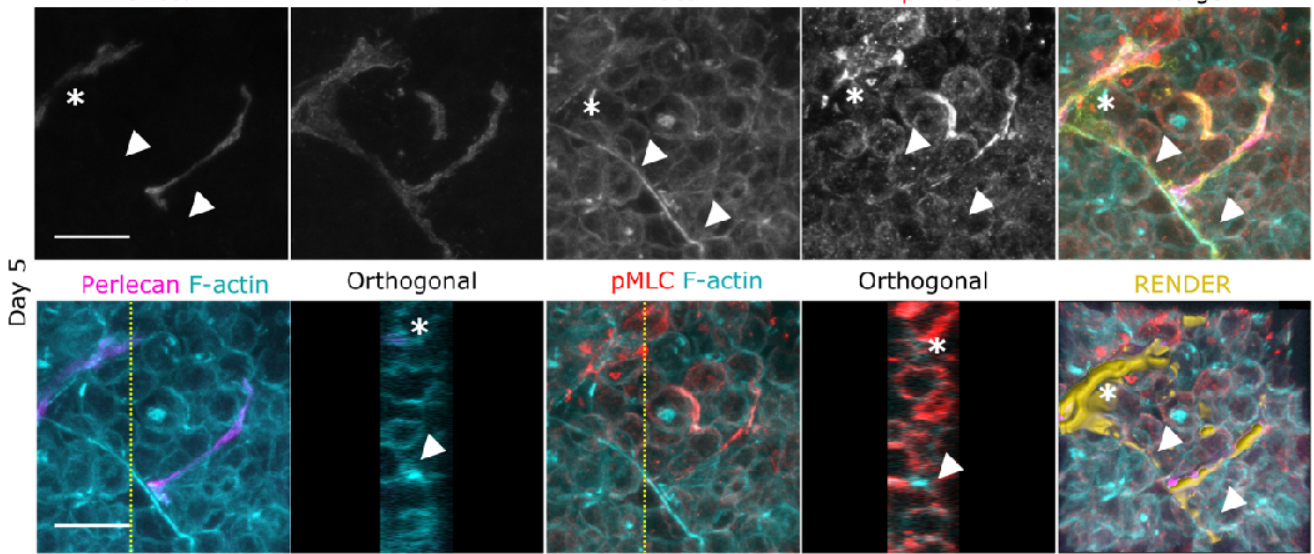

RENDER

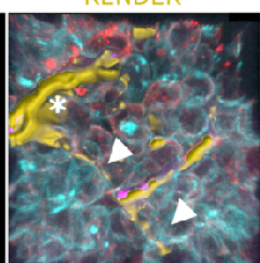


Figure 2 - Actomyosin contractility sets FRC network tension in response to tissue expansion.

A) Lymph node Mass post IFA/OVA immunisation. $\mathrm{N}>5$ B) Recoil curves of network displacement $(\mu \mathrm{m})$ (mean \pm SEM). $\mathrm{N}>5 \mathrm{C}$ ) Initial recoil velocity $\left(\mu \mathrm{m} / \mathrm{s}^{-1}\right)$ post IFA/OVA immunisation, $+/$ - ROCK inhibition (Y27632). Resistive myosin forces (blue text = ratio between PBS/Y27). Box plot indicates median, interquartile range and $\mathrm{min} / \mathrm{max}$. Two-way ANOVA with Tukeys multiple comparisons, $* * p<0.01, * * * * p<0.0001$. N>5. D) PDGFR $\alpha$-mGFP (FRCs) ablation ROI (white box) and cut site (red dotted line). Scale bar $50 \mu \mathrm{m}$. Recoil displacement (white arrowheads) with pre (green) and post cut (magenta) overlay. Scale bar $10 \mu \mathrm{m}$. E) Paracortical FRCS post IFA/OVA immunisation. Perlecan (matrix), PDPN (FRC), phalloidin (F-actin) and pMLC. Asterisk and arrowheads indicate F-actin cables. Orthogonal views (yellow dotted line, YZ axis, $10 \mu \mathrm{m}$ depth). Scale bar $10 \mu \mathrm{m}$. 
bioRxiv preprint doi: https://doi.org/10.1101/2021.05.27.446027; this version posted July 30, 2021. The copyright holder for this preprint (which was not certified by peer review) is the author/funder, who has granted bioRxiv a license to display the preprint in perpetuity. It is made available under ACC-BY-NC-ND 4.0 International license.
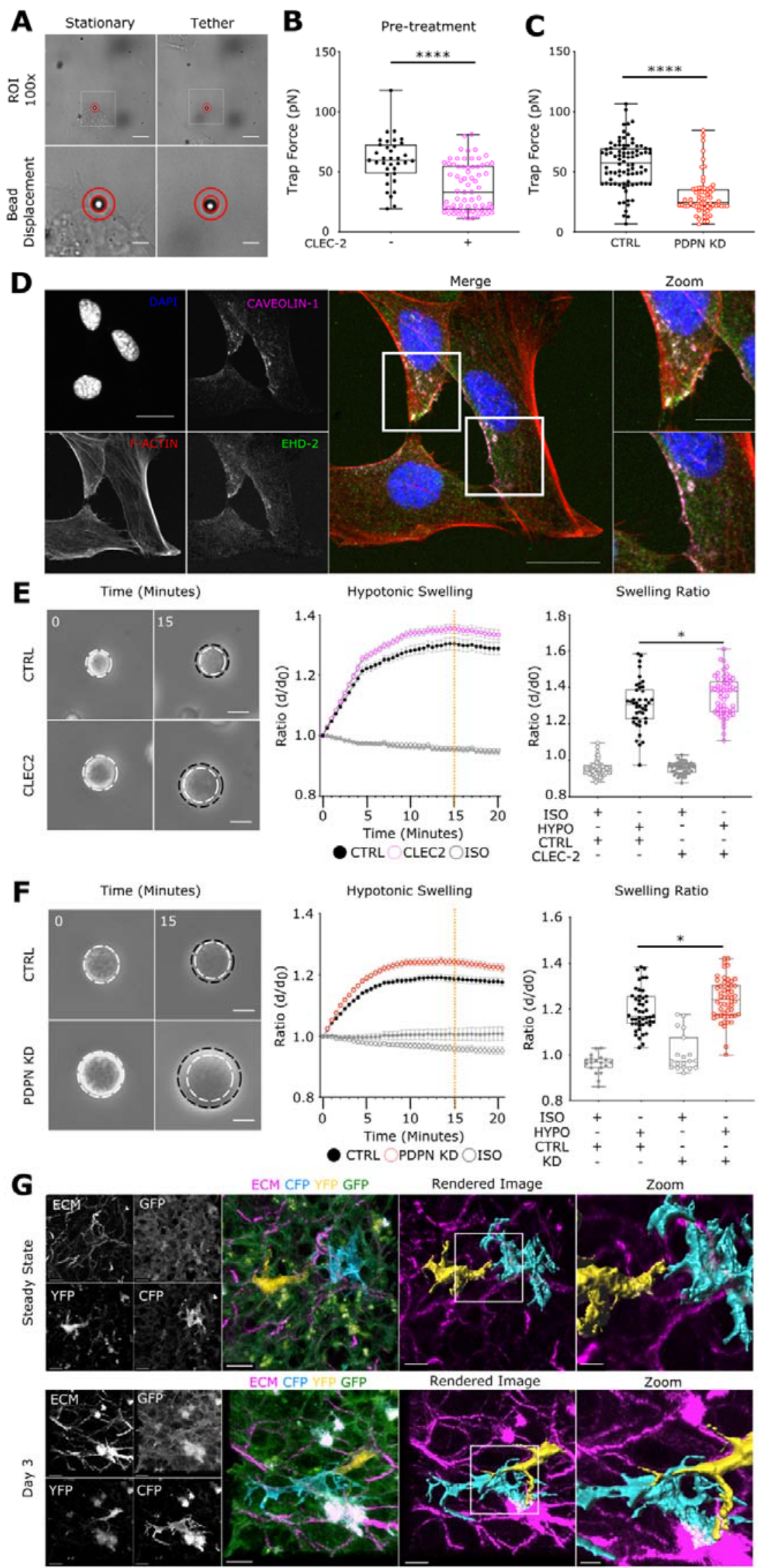


\section{Figure 3 - CLEC-2/PDPN control intrinsic FRC mechanics for morphological adaption}

A) Optical tweezers generate membrane tethers of FRCs, scale bar $10 \mu \mathrm{m}$. Stationery (bottom left) and tether (bottom right) bead displacement (red circles). Scale bar $2 \mu \mathrm{m}$. B) Trap force $(\mathrm{pN} / \mu \mathrm{m})$ of FRCs pre-treated with CLEC-2. Box plot indicates median, interquartile range and min/max. MannWhitney test, $\mathrm{p}<0.001$. Points represent individual cells. $\mathrm{N}>35$. C) Trap force ( $\mathrm{pN} / \mu \mathrm{m})$ of PDPN shRNA KD FRCS Trap force measurement of FRCs. Box plot indicates median, interquartile range and $\min / \max$. Mann-Whitney test, $* * * * p<0.001$. Points represent individual cells. N $>64$. D) Caveolae structures in FRCs, scale bar $20 \mu \mathrm{m}$. DAPI (Blue), F-actin (Red), Caveolin-1 (magenta) and EHD2 (Green). Zooms (white box), scale bar $10 \mu \mathrm{m}$. E) Swelling of PDPN CTRL FRCs +/- pre-treatment CLEC2. Initial size (white circle) post swelling (black circle). Scale bar $25 \mu \mathrm{m}$. Change in diameter ratio (middle, mean \pm SEM). Diameter ratio of control and CLEC2 treatment at 15 minutes post swelling (orange dotted line, (right)). Box plot indicates median, interquartile range and $\mathrm{min} / \mathrm{max}$. One-way ANOVA with Tukeys multiple comparisons, $p<0.01$. N $>42$. F) Swelling of PDPN shRNA KD FRCs. Initial size (white circle) post swelling (black circle). Scale bar $25 \mu \mathrm{m}$. Change in diameter ratio (middle, mean \pm SEM). Diameter ratio of control and CLEC2 treatment at 15 minutes post swelling (orange dotted line, (right)). Box plot indicates median, interquartile range and $\min / \mathrm{max}$. One-way ANOVA with Tukeys multiple comparisons, ${ }^{*} p<0.01 . \mathrm{N}>19$. G) Individually labelled FRCs in vivo, scale bar $10 \mu \mathrm{m}$. Zoomed region (white box), scale bar $5 \mu \mathrm{m}$. 
A

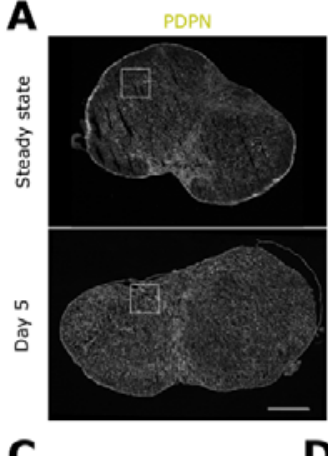

$\mathbf{C}$

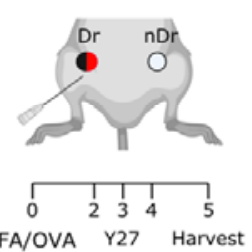

D
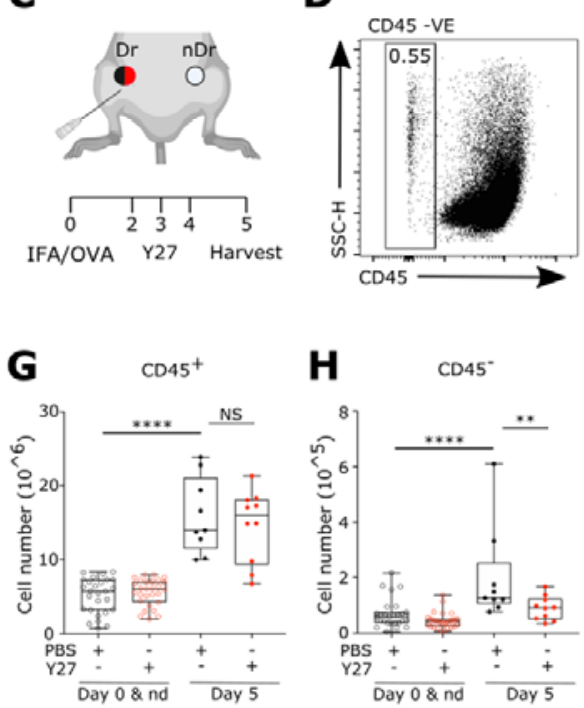
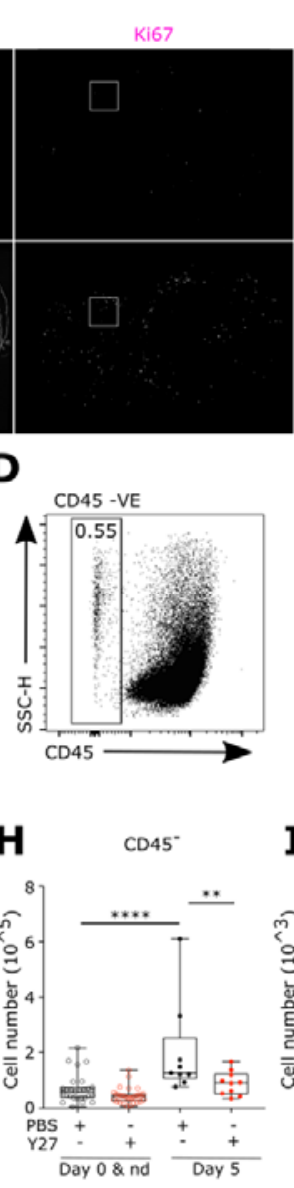

Merge
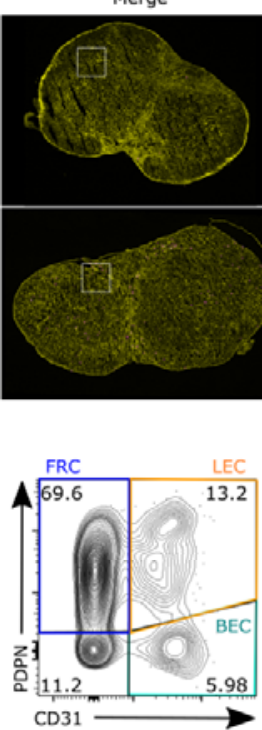

$\mathbf{E}$

I

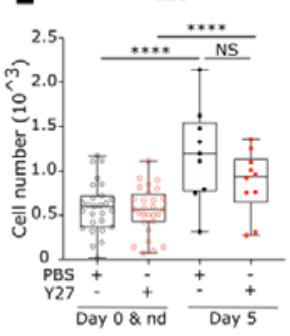

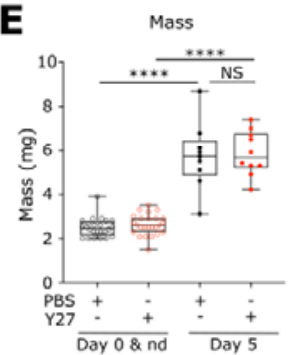

Zoom
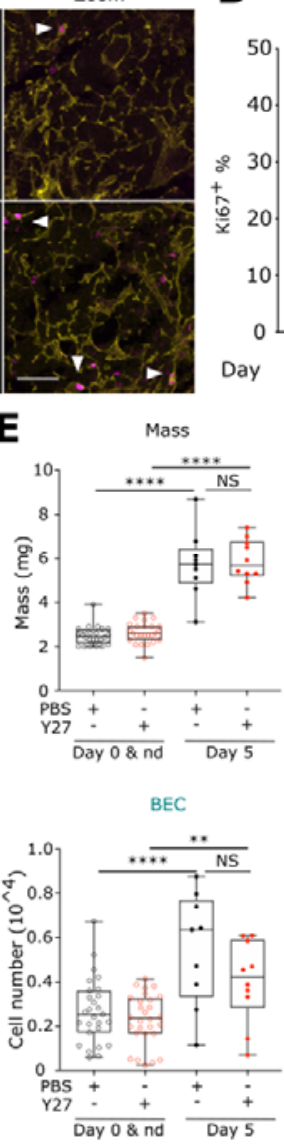

B
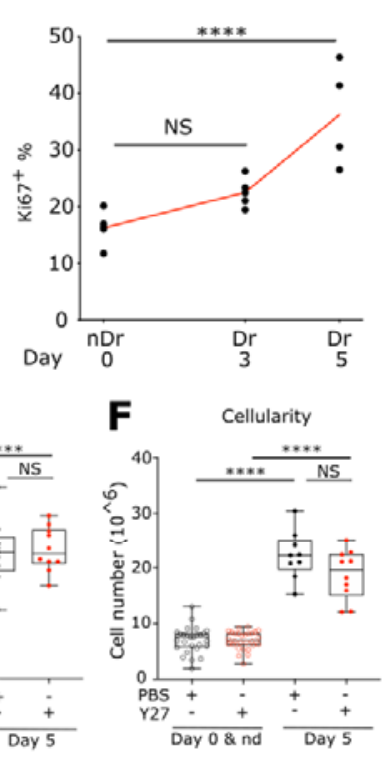

FRC

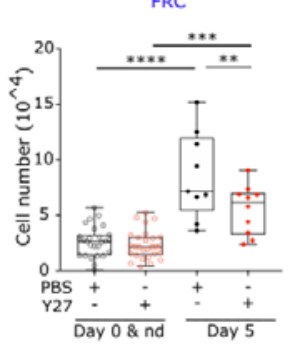

Figure 4 - Mechanical tension gates FRC proliferation

A) LN tile scan of PDPN (left), $\mathrm{Ki}^{+} 7^{+}$(middle). Scale bar $500 \mathrm{~m}$. zoom in T-cell area (white box). Scale bar $50 \mathrm{~m}$. Arrowheads mark PDPN ${ }^{+} \mathrm{Ki}^{+} 7^{+}$FRCs. B) Flow cytometric analysis of Ki67 ${ }^{+} \%$ of $\mathrm{FRC}^{\prime} \mathrm{s}$ post IFA/OVA immunisation. Two-way ANOVA with Tukeys multiple comparisons, ****p<0.0001. N>4. C) Schematic of immunisation and Y27 treatment timeline. D) Flow cytometric gating, representative percentages of CD45- stroma cells (left) and sub-populations (right). Fibroblastic reticular cells (FRC$\mathrm{PDPN}^{+} \mathrm{CD} 31^{\top}$ ), blood endothelial cells $\left(\mathrm{BEC}-\mathrm{PDPN} C D 31^{+}\right)$, lymphatic endothelial cells (LEC$\mathrm{PDPN}^{+} \mathrm{CD} 31^{+}$). E) LN mass. Box plot indicates median, interquartile range and $\mathrm{min} / \mathrm{max}$. Two-way ANOVA with Tukeys multiple comparisons, $* * * * \mathrm{p}<0.0001, * * * \mathrm{p}<0.001$. N $>9$. F-G) Total number of cells post IFA/OVA immunisation, +/- ROCK inhibition (Y27632). Immune cells (CD45 ${ }^{+}$), stroma (CD45 ). I) Total number of LECs (left), BECs (middle) and FRCs (right) post IFA/OVA immunisation, +/- ROCK inhibition (Y27632). Box plot indicates median, interquartile range and $\mathrm{min} / \mathrm{max}$. Two-way ANOVA with Tukeys multiple comparisons, ${ }^{* * * * p<0.0001, ~}{ }^{* * * p<0.001, * * p<0.01 . ~ N>9 .}$ 


\section{Science \\ MIAAAS}

\section{Supplementary Materials for}

Tissue homeostasis and adaptation to immune challenge resolved by fibroblast network mechanics

Harry L. Horsnell ${ }^{1}$, Robert J. Tetley ${ }^{2}$, Henry De Belly ${ }^{3}$, Spyridon Makris ${ }^{1}$, Lindsey J. Millward ${ }^{1}$, Agnesska C. Benjamin ${ }^{1}$, Ewa K. Paluch ${ }^{3}$, Yanlan Mao ${ }^{2,4}$, Sophie E. Acton ${ }^{1 \#}$

Correspondence to: s.acton@ucl.ac.uk

This PDF file includes:

Materials and Methods

Figs. S1 to S5

Tables S1

References (1 to 14)

Captions for Movies S1 to S4

Other Supplementary Materials for this manuscript include the following:

Movies S1 to S4 


\section{Materials \& Methods}

Mice

Experiments were performed in accordance with national and institutional guidelines for animal care and approved by the institutional animal ethics committee review board, European research council and the UK Home office. Breeding of animal lines were maintained off site at Charles River Laboratory. Wild type C57BL/6J mice were purchased from Charles River Laboratories. Novel mouse model of PDGFR $\alpha$-mGFP-CreERT2 generation. EGFP with a membrane tag ( $\mathrm{N}$-terminal 0-20 amino acids of neuromodulin GAP-43) was inserted into the PDGFR $\alpha$ gene locus in combination with a CreERT2 cassette (linked to mGFP with a P2A self cleavage peptide ${ }^{1}$ ) using Cyagen CRISPR Cas9 technology. PDGFR $\alpha$-mGFP-CreERT2 x R26R-Confetti ${ }^{2}$ mice were also generated. Females and males aged between 8-12 weeks were used for experiments, unless stated otherwise.

\section{$\underline{\text { Immunisation Model }}$}

Mice were immunised via subcutaneous injection into the right flank, proximal to the hip, with $100 \mu$ l of an emulsion of Ovalbumin (OVA) with incomplete Freunds adjuvant (IFA) (100 $\mu \mathrm{g}$ OVA per mouse) (Hooke Laboratories). Where stated, mice were treated with Y-27632 (Tocris 1254). 10 $\mu \mathrm{l} \mathrm{of} 1 \mathrm{mg} / \mathrm{ml}$ Y-27632 dissolved in PBS was injected subcutaneously into the right flank, proximal to the hip. $10 \mu \mathrm{l}$ of sterile PBS was used as injection control. Y-27632/PBS injections were given on 3 consecutive days 24 hours post IFA/OVA injection. After 5 or 3 days mice were culled and inguinal lymph nodes (LNs) from both flanks (naïve and inflamed) were extracted for paired histological studies, flow cytometry analysis or ex vivo laser ablation.

\section{Ex vivo cultures}

Preparation for ex vivo lymph nodes laser ablation was optimised following previously established methods ${ }^{3-6}$. UltraPure ${ }^{\mathrm{TM}}$ Low melting point agarose (Thermo Fischer Scientific) prepared in PBS at $3 \% \mathrm{w} / \mathrm{v}$ and maintained at $37^{\circ} \mathrm{C}$. Lymph nodes were embedded into agarose and left for 5 minutes to set on ice. Lymph node blocks were secured by superglue to cutting stage and placed into ice cold PBS cutting chamber. Lecia Vibratome (VT1200S) $200 \mu \mathrm{m}$ thick sections were cut at a rate of $0.3 \mathrm{~mm} / \mathrm{s}$ and $1.5 \mathrm{~mm}$ amplitude until the LN was completely sectioned. Collected sections were placed into RPMI 1640 containing 10\% FBS, 1\% penicillin and streptomycin (P/S) (Thermo Fischer scientific 15140122) and 1\% Insulin-Transferrin-Selenium (ITS) (Thermo Fischer scientific 41400045) at $37^{\circ} \mathrm{C}, 10 \% \mathrm{CO}_{2}$. Sections recovered for an hour before been used for live imaging. 


\section{Laser ablation}

LN sections were transferred to glass bottom 35mm MatTek dishes (P35G-1.5-20-C) with a small volume of RPMI media containing $1 \% \mathrm{P} / \mathrm{S}$ and $1 \%$ ITS. A glass cover slip was placed on top to secure the section. Sections imaged on a Zeiss LSM 880 inverted multiphoton microscope with the imaging chamber maintained at $37^{\circ} \mathrm{C}, 10 \% \mathrm{CO}_{2}$. Where stated, sections were treated with $100 \mu \mathrm{M} \mathrm{Y}-27632$ (Tocris 1254) diluted in ex vivo culture media for at least 1 hour prior to imaging and ablation. Sections were imaged with a Plan Apochromat 40x oil objective (NA1.3), 1024*1024 resolution and $4 x$ digital zoom for ablation ROIs. Laser ablation of the FRC network was achieved by using a pulsed Chameleon Vision II TiSa Laser power $75-80 \%$ (coherent) tuned to $760 \mathrm{~nm}$. Ablation was performed on small, manually defined linear regions of interest between FRC connections away from cell bodies. Ablation was performed in a single z-plane at the centre of the FRC connection in a vibratome slice. Time-lapse videos were recorded over 25 seconds on a single channel (PMT detector) $512 * 512$ pixels, with 521 millisecond scan speed per frame to capture recoil of the network. Recoil of the network was calculated by manually measuring displacement of the FRC network between two points located away from the ablation site, with initial recoil velocities calculated from the displacement one frame after the cut, as in other studies ${ }^{7,8}$.

\section{Immunostaining of tissue sections}

LNs that were used for sectioning and immunofluorescence were fixed in Antigen fix (DiaPath) for 2 hours at $4^{\circ} \mathrm{C}$ with gentle agitation. LNs were washed for 30 minutes in PBS before being applied to $30 \% \mathrm{w} / \mathrm{v}$ sucrose $0.05 \%$ sodium azide solution at $4^{\circ} \mathrm{C}$ overnight. LNs were dipped into Tissue-Tek OCT before being embedded into moulds containing OCT. A maximum of six lymph nodes were embedded into a single block for comparative analysis. Lymph nodes were sectioned on the Leica cryostat at a thickness of $20 \mathrm{um}$.

For immunofluorescent staining sections were permeabilised and blocked using $10 \%$ Normal goat serum (NGS), $0.3 \%$ Triton X-100 in PBS for two hours at room temperature. Primary antibodies were prepared by centrifugation at $13,000 \mathrm{rpm}$ for 5 minutes at $4^{\circ} \mathrm{C}$. Primary antibodies were diluted according to Antibody Table 1 in 10\% NGS, 0.01\% Triton X-100 in PBS. Sections were incubated overnight at $4^{\circ} \mathrm{C}$. Sections were then brought to room temperature and washed 3 times for 15 minutes each in $0.05 \%$ PBS-Tween 20 . Sections were then blocked using $10 \%$ Normal goat serum (NGS), $0.3 \%$ Triton X-100 in PBS for two hours at room temperature. Secondary antibodies were then prepared similar to primary antibodies with dilutions in Antibody Table 1. Secondary antibodies were applied to sections for $2 \mathrm{hrs}$ at room temperature. This was followed by two 15 -minute washes 
of $0.05 \%$ PBS-Tween 20 and a final wash of 15 minutes in PBS. Sections were then mounted using mowiol mounting media.

For staining of $200 \mu \mathrm{m}$ thick vibratome sections, slices were first fixed in Antigen fix (DiaPath) for 2 hours at $4^{\circ} \mathrm{C}$ with gentle agitation. Sections were placed into $0.1 \mathrm{M}$ Tris-HCL pH7.4 on ice for 30 minutes. Sections were permeabilised using IHC buffer containing $0.5 \%$ BSA, 2\% Triton X-100 (Sigma-Aldrich) in $0.1 \mathrm{M}$ Tris-HCL pH7.4 for 20 minutes at $4^{\circ} \mathrm{C}$ with gentle agitation. Primary antibodies were prepared by centrifugation at $13,000 \mathrm{rpm}$ for 5 minutes at $4^{\circ} \mathrm{C}$. Primary antibodies were diluted into IHC buffer, according to Antibody Table 1, and applied to the sections for $1 \mathrm{hr}$ at $4^{\circ} \mathrm{C}$ with gentle agitation. Sections were then washed twice with $0.1 \mathrm{M}$ Tris-HCL for 15 minutes. Secondary antibodies were then prepared similar to primary antibodies with dilutions in Antibody Table 1. Secondary antibodies were applied to sections for $1 \mathrm{hr}$ at $4{ }^{\circ} \mathrm{C}$ with gentle agitation. Two final washes of $0.1 \mathrm{M}$ Tris-HCL were performed before mounting the sections with mowiol mounting media and a glass coverslip. Sections $(15-3=40 \mu \mathrm{m})$ were then imaged on the Zeiss LSM 880 inverted multiphoton microscope using a Plan Apochromat 40x oil objective (NA1.3).

Unless otherwise stated confocal images were acquired using Leica TCS SP8 STED 3X or the Leica TCS SP5. Images were captured at $1024 * 1024$ pixels, 3 line average onto HyD or PMT detectors. Fluorophore excitation and acquisition was performed in a sequential and bidirectional manner. Imaging regions were manually defined and z-stacks $(15-40 \mu \mathrm{m})$ with regular z-intervals ranging from 0.4um or 1um (dependent on sample) were acquired using a motorised stage. Tile scans were automatically stitched (numerical) using Leica imaging software.

\section{Flow cytometry of lymph nodes}

LNs were carefully dissected, weighed, and placed into RPMI 1640 media on ice. LNs were then processed as previously described ${ }^{9,10}$. Briefly, LNs were placed into a digestion buffer containing collagenase D $(250 \mu \mathrm{g} / \mathrm{ml})$ (Millipore Sigma), dispase II $(800 \mu \mathrm{g} / \mathrm{ml})$ (Thermo Fisher Scientific) and DNase I (100 $\mu \mathrm{g} / \mathrm{ml}$ ) (Sigma Aldrich). LNs were gently digested in a water bath at $37{ }^{\circ} \mathrm{C}$, removing and replacing the cell suspension every 10 minutes until completely digested. Cell suspensions were then centrifuged at $350 \mathrm{~g}$ for 5 minutes. The cells were resuspended into flow cytometry buffer (FACS) consisting of $1 \%$ BSA (Sigma Aldrich), 5mM EDTA (Sigma Aldrich) in PBS, filtered, counted, and resuspended at $10 \times 10^{6} \mathrm{cells} / \mathrm{ml} .2 .5 \times 10^{6}$ cells were then plated into 96 well plates for surface and intracellular staining of a stromal cell panel (Antibody Table 1). Cells were blocked with $\mathrm{CD} 16 / \mathrm{CD} 32$ Mouse $\mathrm{Fc}$ block (BD) and then stained with primary antibodies for 20 minutes at $4^{\circ} \mathrm{C}$. For 
intracellular staining of Ki67 cells were fixed and permeabilised using FOXP3 fix/perm buffer as specified by the manufacturer (BioLegend). Samples were run on the Fortessa X20 flow cytometer (BD Biosciences) at the UCL Cancer Institute. Data was analysed using Flow Jo software (FlowJo, LLC).

\section{Cell lines}

Immortalised fibroblastic reticular cells were generated as described in Acton et al $2014^{10,11}$. Parental immortalised fibroblastic reticular cell line (Control FRC). Podoplanin (PDPN) was stably knocked down (PDPN KD FRC) in the parental cell line by transfection of a PDPN shRNA lentivirus. PDPN was completely depleted from the parental cell line (PDPN KO FRC) using CRISPR cas9 genetic deletion. In all experiment where exogenous PDPN mutant cell lines (PDPN WT, PDPN T34A, PDPN S167A-S171A) were used, protein production was induced by the addition of $1 \mu \mathrm{g} / \mathrm{ml}$ of doxycycline for 48 hours. Cell lines were maintained at $10 \% \mathrm{CO}_{2}, 37^{\circ} \mathrm{C}$ in Dulbecco's modified eagle medium (DMEM) (Thermo Fischer scientific) supplemented with $10 \% \mathrm{FBS}, 1 \% \mathrm{P} / \mathrm{S}$ and $1 \%$ ITS unless otherwise stated. Cells were treated with recombinant CLEC2-FC or Control-FC supernatant ${ }^{10,11}$ for approximately $2 \mathrm{hrs}$, where indicated, to assay the effect of CLEC-2 signalling through PDPN. Figure $3 C$ is a paired analysis of the same cell before and 15 minutes after CLEC-2 treatment.

\section{Tether pulling and trap force measurements}

Trap force measurements were performed using a home built optical tweezer using a 4W $1064 \mathrm{~nm}$ laser quantum Ventus within a 100x oil immersion objective (NA 1.30, CFI Plan Fluor DLL, Nikon) on an inverted microscope (Nikon Eclipse TE2000-U) equipped with a motorised stage (PRIOR Proscan). The optical tweezer was calibrated following previous studies ${ }^{12,13}$. The trap force calibration was performed in every experiment with typical calibration trap stiffness of $\mathrm{k} \sim 0.114 \mathrm{pN} / \mathrm{nm}$. Measurements were performed using concanavalin-A coated $(50 \mathrm{ug} / \mathrm{ml}$ ) carboxyl latex beads, (1.9um diameter, Thermo Fischer). Beads were incubated on a shaker with concanavalin-A for two hours prior to experiment. Beads were applied to the culture media, manipulated by the optical trap and brought into contact with the cellular membrane and typically held for 2-5 seconds to allow binding to membrane. Bead position was recorded every 90 milliseconds in bright field prior and during tether formation. Cells were maintained in the trap at 37 degrees and had $\mathrm{CO}_{2}$ flowing into the chamber. Addition of CLEC-2 or CTRL supernatant was 2-4 hours prior to measurement of trap force. Trap force $\left(\mathrm{F}_{\mathrm{t}}-p N / \mu m\right)$ was then calculated based on the trap calibration $(k)$, bead position $(\Delta x)$ using a home-made Fiji macro ${ }^{13}$ and the equation $F_{T}=k \Delta x$. 


\section{Omsotic swelling assay}

Osmotic shock was performed in accordance with previously reported protocols ${ }^{14}$. By altering the osmolarity of a solution cells will swell or shrink. Osmolarity was estimated using osmolarity calculations. Isotonic solution was prepared with $137 \mathrm{mM} \mathrm{NaCl}, 5.4 \mathrm{mM} \mathrm{KCl}, 1.8 \mathrm{mM} \mathrm{CaCl} 2,0.8 \mathrm{mM}$ $\mathrm{MgCl}_{2}, 20 \mathrm{mM}$ HEPES, 20mM D-glucose and $\mathrm{pH}$ to 7.4 with $\mathrm{NaOH}$. Hypotonic (Hypo) solutions were prepared by diluting the isotonic preparation in milliq water i.e. $50 \mathrm{mOsm}$ is a $1 / 6$ dilution of Iso. To control for the dilution of ions in solution and account for the effect this may have on swelling, the 50 mosm solution was restored to $330 \mathrm{mOsm}$ using D-mannitol at $280 \mathrm{mM}$, acting as the true isotonic control (Iso). Cells were dissociated from cell culture with Sterile Dulbecco's phosphate buffered saline (DPBS) (Thermo Fischer scientific) + 2mM EDTA and placed onto individual 35mm MatTek dishes and allowed to settle for 30 minutes. After 30 minutes the cells remain rounded on the coverslip. Cells were then treated with either Iso, Hypo 50mOsm or Extreme Hypo OmOsm for one hour. Phase contrast images of cell swelling were captured every 30 seconds using 20x air objective on a Nikon Ti inverted microscope with a motorised stage controlled by NIS-elements software. Diameter of individual swelling cells were calculated using manual circular ROIs. The ratio of swelling was then calculated by dividing all diameters (d) by the initial diameter (d0). Area under the curves were calculated for the first 20 minutes of the swelling response.

\section{Western blotting}

Equal cell numbers were grown to confluency and protein was isolated using $300 \mu \mathrm{l}$ of $4 x$ Laemmlli Lysis Buffer (Bio-Rad) and cell lifters (Fisher Scientific). Lysates were then sonicated for 20 seconds followed by 10 minutes at $95^{\circ} \mathrm{C} .1 \% \beta$-mercaptoethanol ( $143 \mathrm{mM}$ stock, Sigma Aldrich) was applied to samples to reduce oligomerised protein structures. Electrophoresis gels were loaded with the same quantity of lysates and run for 60 minutes at 110v. Transfer to PDVF membranes were carried out at $65 \mathrm{v}$ for $2 \mathrm{hr}$ at 4 . Membranes were blocked for $2 \mathrm{hrs}$ at room temperature with $5 \%$ skim milk powder (Sigma-Aldrich), 1\% BSA in TBS and stained with primary antibodies (Antibody Table 1) overnight at 4 in 1:5 diluted blocking buffer. Membranes were then washed thoroughly with TBS 0.05\% Tween 20 and incubated with HRP-conjugated secondary antibodies (Antibody Table 1) in 1:5 blocking buffer. After washing with TBS $0.05 \%$ Tween 20 . The membranes were visualised using ECLHRP reaction and imaged using Image Quant 5000 (GE Lifesceinces).

Linear unmixing and Imaris rendering

Imaging of PDGFR $\alpha$-mGFP CreERT2 Confetti LN sections was carried out using lambda mode and chameleon laser at 900um to acquire multi-channel images. Widefield images and z-stack intervals 
of $0.5 \mathrm{~mm}$ were obtained, for an approximate thickness of 30-60uM. The emission wavelengths for each fluorophore were set on Zeiss zen black software spectral unmixing function by selecting labelled cells within the confetti imaging. The 2 nd harmonic of the two-photon laser detected the ECM conduit structure in the lymph node. Rendering of PDPN, CFP and YFP in Fig. 1, 2, 5, Fig. S5 was achieved using Imaris surface tools.

\section{Quantification and statistical analysis}

Prism7 Software (GraphPad) was used to perform multiple statistical analyses including appropriate tests were performed as indicated in figure legends. In general, comparison of multiple groups was performed using Two-way ANOVA with Tukey's multiple comparisons or One-way ANOVA with Kruskal-Wallis test depending on the distribution of the data. Comparisons of two data sets were most performed using Two-tailed Mann-Whitney tests. 


\section{Figs. S1 to S5}
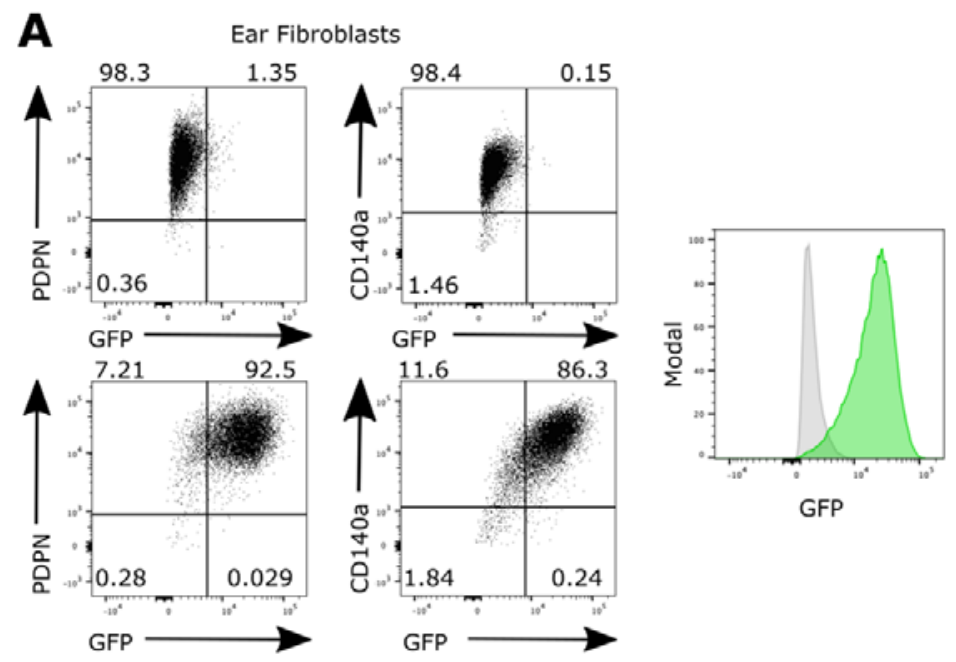

Fig. S1 - Flow cytometry of PDGFR -mGFP fibroblasts

A) Flow cytometry panel of PDPN ${ }^{+}$and $\mathrm{CD} 140{ }^{+}$skin fibroblasts expressing GFP. Top two panels show GFP- control fibroblasts. Bottom two panels show PDPN+, GFP+ and CD140a+ GFP+ fibroblasts. Right panel shows control fibroblasts (grey) to PDGFR -mGFP fibroblasts (green). 

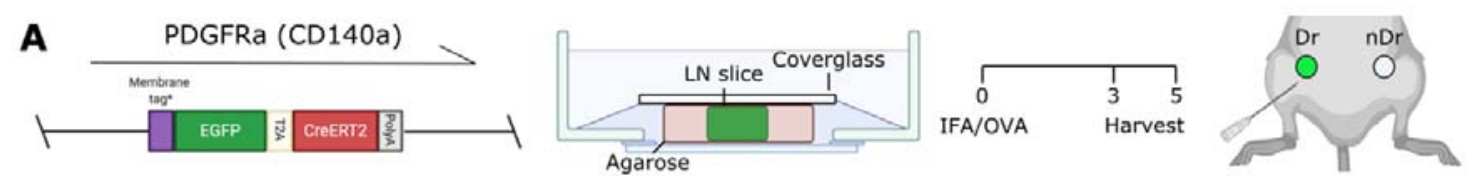

B

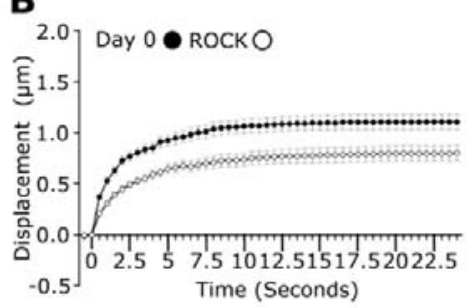

C
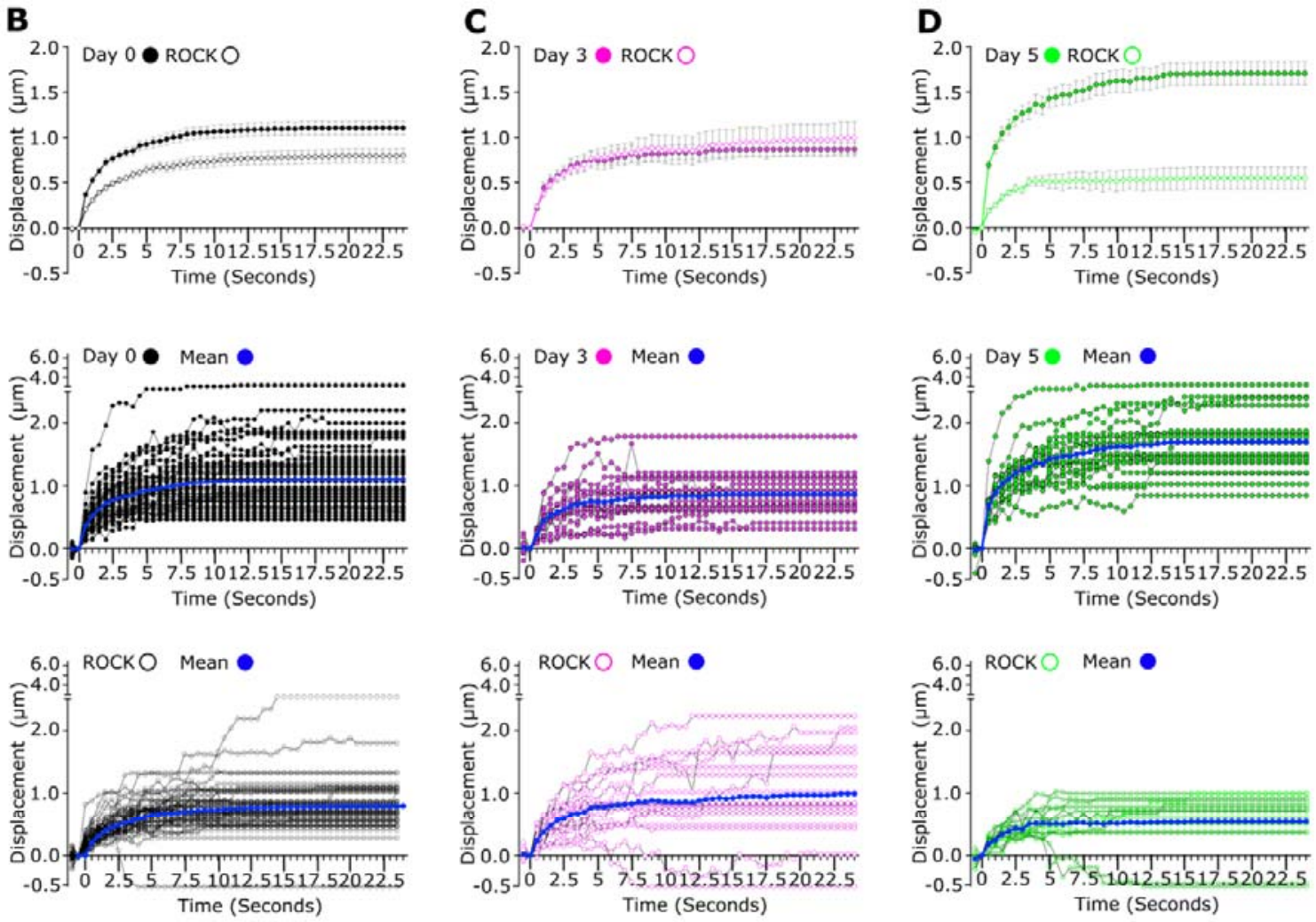

E
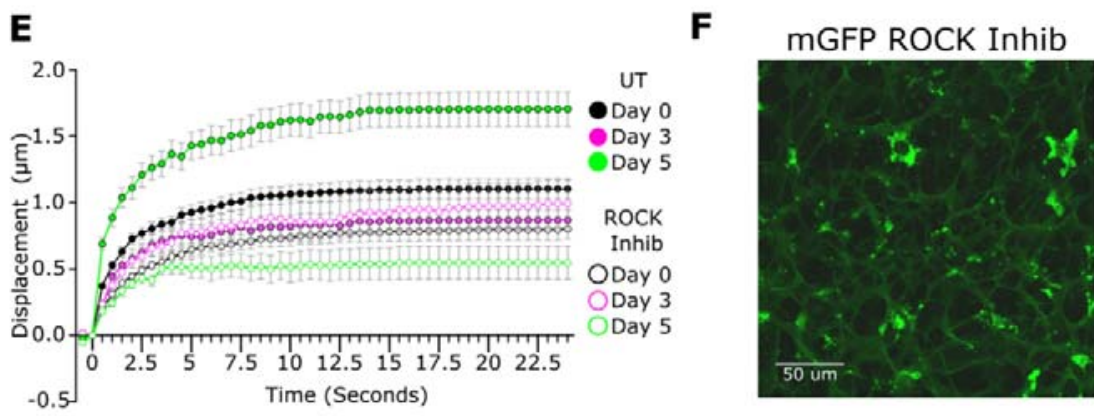

\section{Fig. S2 - Y27 treatment of lymph node slices reduce tension in the FRC network}

A) Schematic of immunisation and animal models. A membrane targeted GFP molecule is driven under the (PDGFR ) promoter (left). LN nodes were embedded in low melt agarose and sliced at $200 \mathrm{~m}$ thickness before being secured by cover glass for imaging (middle). IFA/OVA is used as model immunisation with inguinal draining (dr) and non-draining ( $\mathrm{nDr}$ ) lymph nodes ( $\left(\mathrm{LN}^{\prime} \mathrm{s}\right)$ harvested day 3 or day 5 post immunisation (right). B-D) Recoil curves show displacement over time (mean \pm SEM) (top panel). Individual recoil curves of control (middle) and Y27 treated LNs (bottom). N>5 animals per condition. E) Recoil curves show displacement over time (mean \pm SEM), comparing untreated and Y27 treated LNs. Blue shading indicates initial recoil phase. $N>5$ animals per condition. F) Membrane GFP visualises the FRC network in the ex vivo lymph node slice. Y27 treatment has no effect on the FRC network connectivity. Scale bar $50 \mathrm{~m}$. 


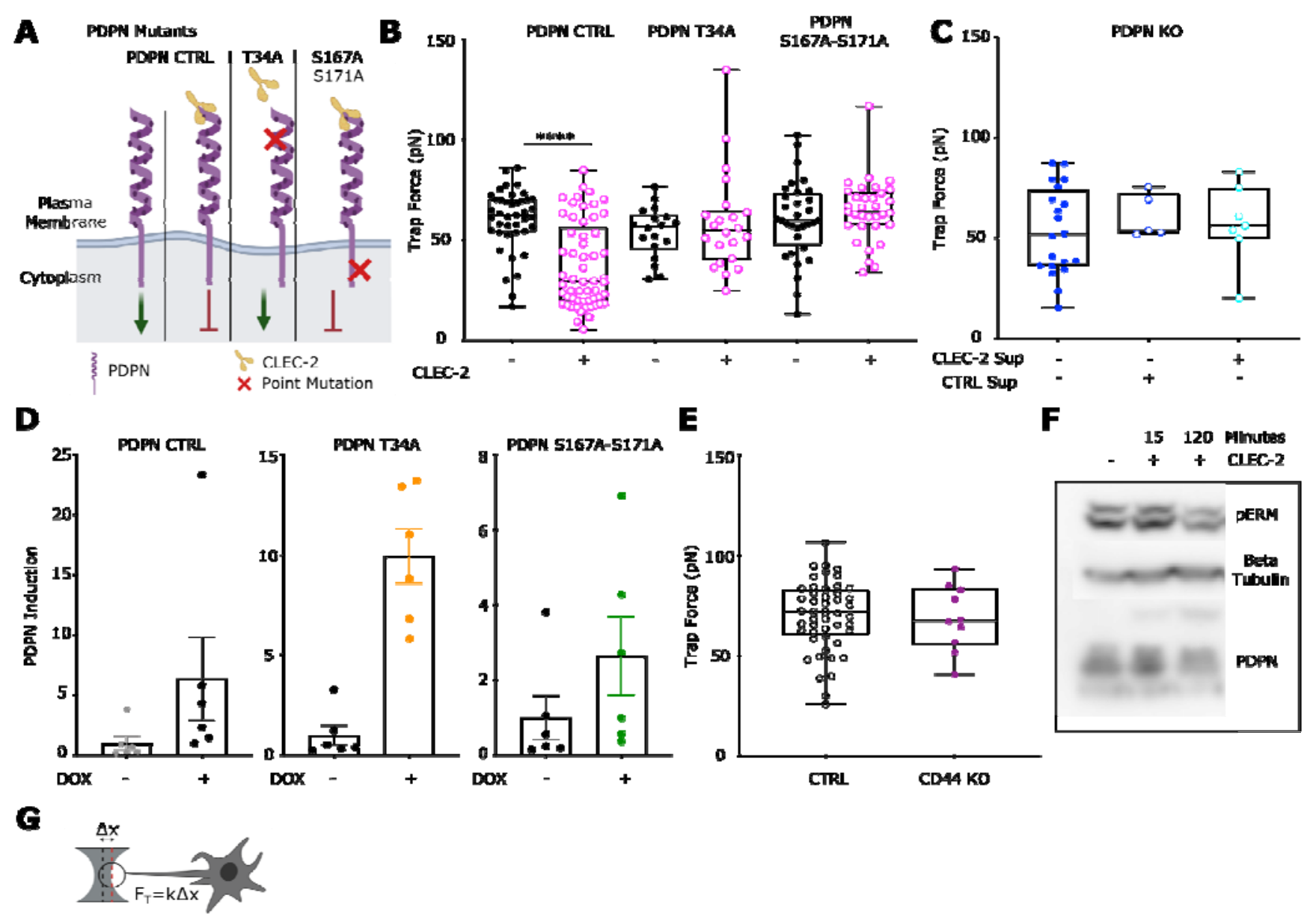

Fig. S3 - Induction of PDPN mutants in PDPN KO fibroblastic reticular cell line

A) Schema of exogenous PDPN mutants and interaction with CLEC2. Green arrow denotes active signalling by PDPN leading to actomyosin contractility, red arrow indicates inhibition of PDPN signalling and reduction in actomyosin contractility. B) Trap force measurements of FRCs expressing PDPN mutants after pre-treatment of CLEC2. Box plots indicates median and interquartile range. Two-way ANOVA with Tukeys multiple comparisons, $p<0.001$. N $>18$. C) Trap force measurements of PDPN CRISPR KO FRCs treated with CTRL or CLEC2 supernatant. One-way ANOVA with Tukeys multiple comparisons. N>5. D) Fold induction of exogenous PDPN, based on PDPN staining geometric mean, for each PDPN mutant cell line treated with or without doxycycline. PDPN CTRL (left), PDPN T34A (middle) and PDPN DSS (right). N=6. E) Trap force measurements of CTRL and CD44 (purple) KO FRCs. Mann-Whitney test, $p<0.001$. Each point represents one cell. $N>10$. F) Representative western blot of pERM in CTRL FRCS after treatment with CLEC2. G) Schema and equation to calculate trap force $\left(F_{t}\right)$ from displacement. 


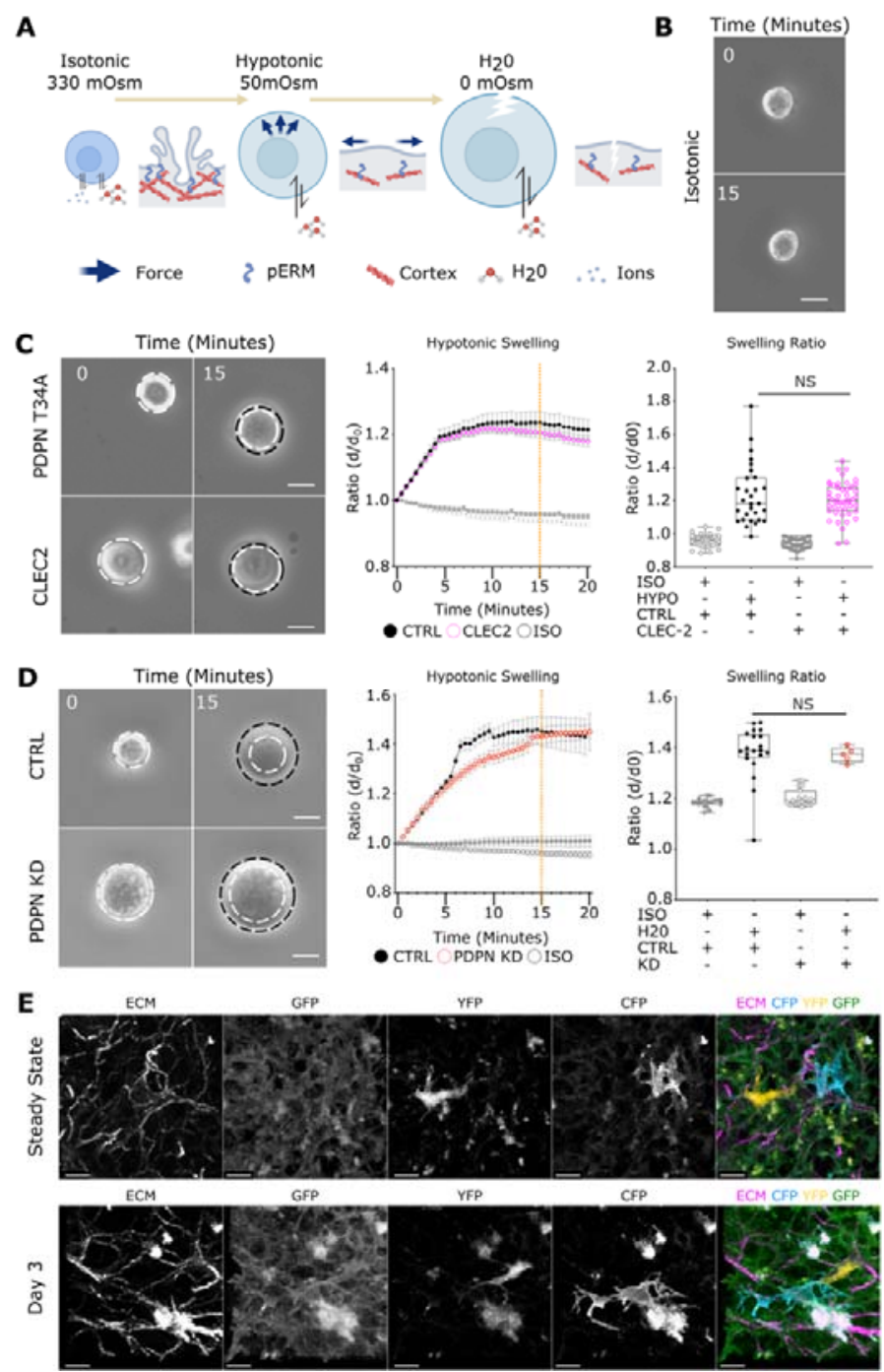

Fig. S4 - Membrane access of fibroblastic reticular cells is increased through the CLEC2/PDPN signalling axis

A) Schematic of osmotic shock experiment, inducing swelling by altering osmolarity. B) Representative stills of FRC in isotonic control media. Scale bar $25 \mathrm{~m}$. C) Time course of PDPN T34A FRCs with or without CLEC2 treatment in hypotonic conditions. Stills (top) show swelling of cells in hypotonic media. White dotted circle marks initial size before swelling and is compared to swelling at $\mathrm{t}=20$ (black dotted circle). Scale bar $25 \mathrm{~m}$. Change in diameter ratio over time (bottom left, mean \pm SEM). Swelling ratio comparisons between control and CLEC2 treatment at 15 minutes post swelling (orange dotted line, bottom right). Box plots indicates median and interquartile range. Oneway ANOVA with Tukeys multiple comparisons. $\mathrm{N}>29$. D) Time course of PDPN CTRL vs PDPN shRNA KD FRCs in extreme hypotonic conditions $\left(\mathrm{H}_{2} \mathrm{O}\right)$. Stills (top) show swelling of cells in hypotonic media. White dotted circle marks initial size before swelling and is compared to swelling at $t=20$ (black dotted circle). Scale bar $25 \mathrm{~m}$. Change in diameter ratio over time (bottom left, mean \pm SEM). Swelling ratio between control and PDPN shRNA KD FRCs at 15 minutes post swelling (orange dotted line, bottom right). Box plots indicates median and interquartile range. One-way ANOVA with Tukeys multiple comparisons. $N>16$. E) Single channel stills of PDGFR -mGFP confetti FRC network in the steady state and day 3 post immunisation. Scale bar $10 \mathrm{~m}$. 
A
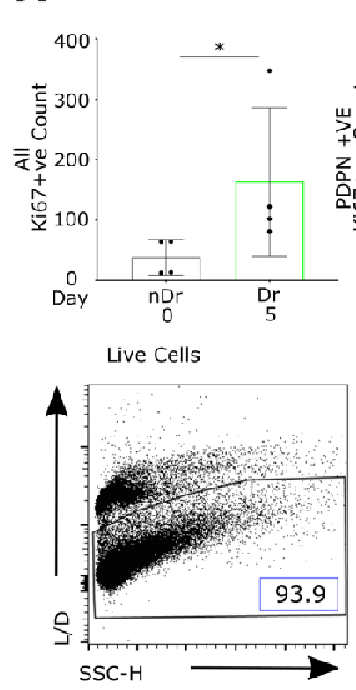

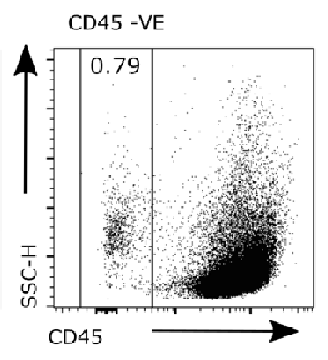

B

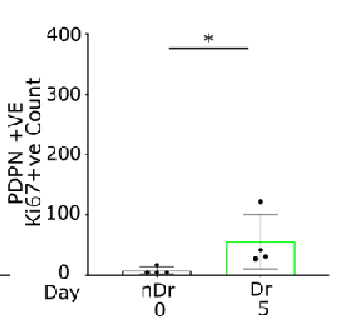

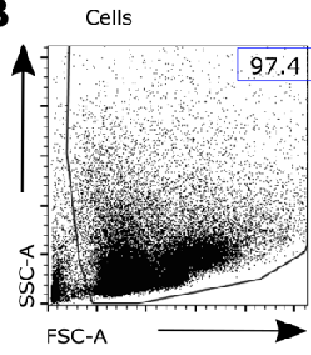

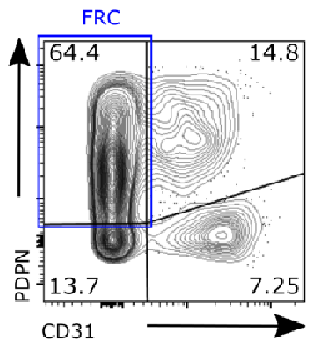

PBS Treated
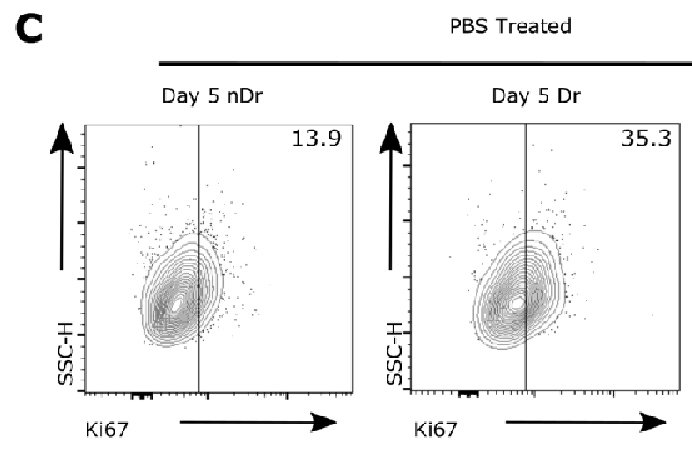

Y27 Treated
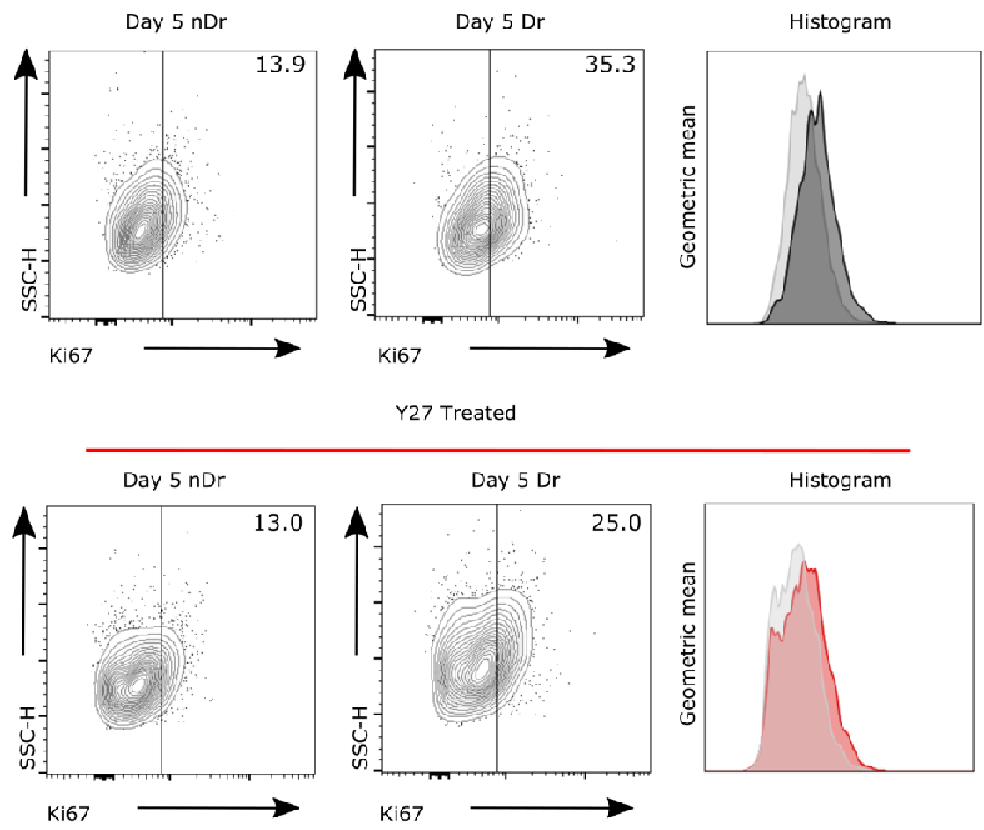

Singlets_1
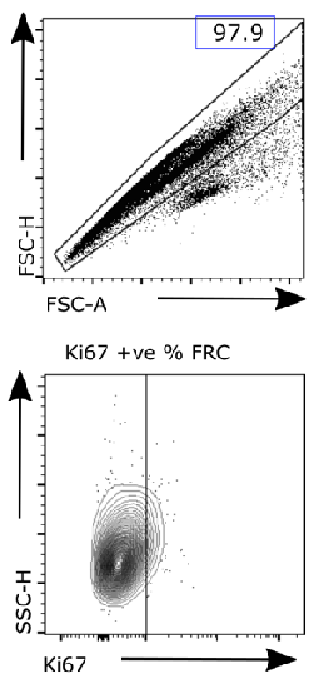

Singlets 2

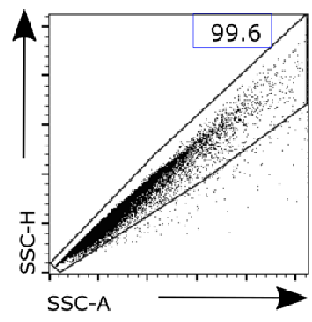

Ki67 FMO

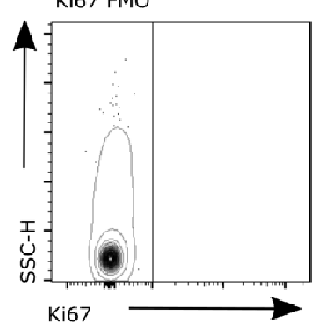

D

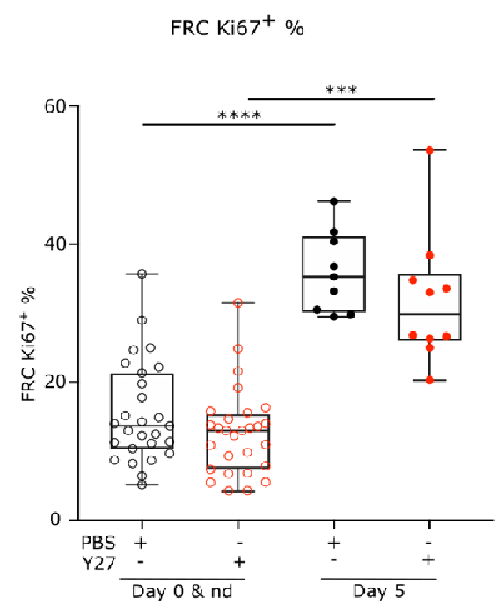

Fig. S5 - Flow cytometry gating strategy and Ki67 measurements

A) Quantification of $\mathrm{Ki}^{+} 7^{+}$cells (top) and $\mathrm{PDPN}^{+} \mathrm{Ki}^{+} 7^{+}$cells (bottom) per $\mathrm{LN}$ region. Box plots indicates median and interquartile range. Mann-Whitney test, $p<0.05$. $N=2$. B) Schema of flow cytometry gating strategy for measurement of stromal cell populations. C) Example of Ki67 ${ }^{+}$FRCs cell plots and histogram of Ki67 geometric mean, comparing immunisation and Y27 treatments. D) Quantification of Ki67 $7^{+}$FRCs. Box plots indicates median and interquartile range. Two-way ANOVA with Tukey's multiple comparisons. ${ }^{* * *} \mathrm{p}<0.0001,{ }^{* * *} \mathrm{p}<0.001$. $\mathrm{N}>9$ animals per condition 
bioRxiv preprint doi: https://doi.org/10.1101/2021.05.27.446027; this version posted July 30, 2021. The copyright holder for this preprint (which was not certified by peer review) is the author/funder, who has granted bioRxiv a license to display the preprint in perpetuity. It is made available under aCC-BY-NC-ND 4.0 International license.

Tables S1

\begin{tabular}{|c|c|c|c|c|}
\hline Antibody & Species & Dilution & Manufacturer & Product ID \\
\hline Alexa Fluor 405 & Donkey anti-rat & $1: 500(I F)$ & abcam & ab175670 \\
\hline Alexa Fluor 488 & Goat-Anti Rabbit & $1: 500(\mathrm{IF})$ & Thermo Fischer Scientific & A-11034 \\
\hline Alexa Fluor 488 & Goat-Anti Hamster & $1: 500(\mid F)$ & Thermo Fischer Scientific & A-21110 \\
\hline Alexa Fluor 555 & Goat-Anti Rabbit & $1: 500(\mathrm{~F})$ & Thermo Fischer Scientific & A-21429 \\
\hline Alexa Fluor 594 & Donkey-Anti rabbit & $1: 500(\mid F)$ & Abcam & ab150076 \\
\hline Alexa Fluor 633 Phalloidin & Amanita phalloides & $1: 200(I F), 1: 500(I F)$ & Thermo Fischer Scientific & A-22284 \\
\hline Alexa Fluor 647 & Goat-Anti Rabbit & $1: 500(\mid F)$ & Thermo Fischer Scientific & A-21245 \\
\hline Alexa Fluor 647 & Goat-Anti Hamster & $1: 500(\mid F)$ & Thermo Fischer Scientific & a-21451 \\
\hline Alexa Fluor 647 & Goat-Anti Rat & $1: 500(\mathrm{IF})$ & Thermo Fischer Scientific & A-21247 \\
\hline Anti-aggrus (Podoplanin) clone $8 \mathrm{~F} 11$ & Rat anti mouse & $1: 1000(W B)$ & MBL international & D190-3 \\
\hline APC/Fire 750 cd31 [390] & Anti-mouse & 1:100 (Flow) & BioLegend & 102434 \\
\hline Brilliant Violet 750TM CD45 & Anti-mouse & 1:100 (Flow) & BioLegend & 103157 \\
\hline BV421 MAdCAM-1 Clone MECA & Rat anti-mouse & 1:100 (Flow) & BD & 742812 \\
\hline Caveolin-1 & Rabbit & $1: 1000(I F)$ & Abcam & ab2910 \\
\hline DAPI & & $1: 1000(\mathrm{~F})$ & Sigma Aldrich & D9542-1MC \\
\hline EHD2 & Rabbit & $1: 500(\mid F)$ & Abcam & ab23935 \\
\hline Histone $\mathrm{H} 3$ & Mouse & $1: 2000(W B)$ & Abcam & 24834 \\
\hline HRP anti mouse & Rabbit anti mouse & $1: 1000$ & Abcam & ab6728 \\
\hline HRP anti Rabbit & Goat anti rabbite & $1: 15000$ (WB) & Abcam & ab6721 \\
\hline HRP anti Rat & Rabbit anti Rat & $1: 5000(\mathrm{WB})$ & Abcam & ab6734 \\
\hline Ki 67 PE Cy7 Clone B56 & anti-mouse & 1:100 (Flow) & BD & 561283 \\
\hline Ki67 & Rabbit & $(1: 200)(\mathrm{F})$ & Abcam & ab16667 \\
\hline PDPN & Hamster & $1: 500(\mid F)$ & Acris & DM3501 \\
\hline PE-CF594 CD140a Clone APA5 (RUO) & Rat anti-mouse & 1:100 (Flow) & $\mathrm{BD}$ & 562775 \\
\hline Perlecan AL76 & Rat anti-mouse & $1: 100(\mid F)$ & Santa Cruz Biotechnology & sc-33707 \\
\hline Phospho-Ezrin(Thr567)/Radixin(Thr564)/Moesin(Thr558) (3141l) & Rabbit & $1: 5000(\mathrm{WB})$ & Cell signalling technologies & $3141 \mathrm{~L}$ \\
\hline Phospho-myosin light chain 2 (Ser 19) & Rabbit & $1: 30(I F)$ & Cell signalling technologies & $3671 \mathrm{~L}$ \\
\hline Podoplanin eFlour 660 & Anti-mouse & $1: 100$ (Flow) & eBioscience & $50-5381-82$ \\
\hline Zombie Aqua Fixable Viability Kit & & 1:1000 (Flow) & BioLegend & 423102 \\
\hline
\end{tabular}

Table S1 - Antibody reagents and dilutions 


\section{References}

1. Liu, Z. et al. Systematic comparison of 2A peptides for cloning multi-genes in a polycistronic vector. Sci. Rep. 7, (2017).

2. Snippert, H. J. et al. Intestinal crypt homeostasis results from neutral competition between symmetrically dividing Lgr5 stem cells. Cell 143, 134-144 (2010).

3. Bajénoff, M. et al. Stromal Cell Networks Regulate Lymphocyte Entry, Migration, and Territoriality in Lymph Nodes. Immunity 25, 989-1001 (2006).

4. Bozdag, E. Intravital Imaging of Dynamic Bone and Immune Systems. Ethics and Information Technology vol. 1763 (2018).

5. Groff, B. D., Kinman, A. W. L., Woodroof, J. F. \& Pompano, R. R. Immunofluorescence staining of live lymph node tissue slices. J. Immunol. Methods 464, 119-125 (2019).

6. Belanger, M. C. et al. Acute lymph node slices are a functional model system to study immunity ex vivo. doi:10.1101/865543.

7. Mao, Y. et al. Differential proliferation rates generate patterns of mechanical tension that orient tissue growth. EMBO J. 32, 2790-2803 (2013).

8. Duda, M. et al. Polarization of Myosin II Refines Tissue Material Properties to Buffer Mechanical Stress. Dev. Cell 48, 245-260.e7 (2019).

9. Fletcher, A. L. et al. Reproducible isolation of lymph node stromal cells reveals sitedependent differences in fibroblastic reticular cells. Front. Immunol. 2, (2011).

10. Acton, S. E. et al. Dendritic cells control fibroblastic reticular network tension and lymph node expansion. Nature 514, 498-502 (2014).

11. De Winde, C. M. et al. Fibroblastic Reticular Cell Response to Dendritic Cells Requires Coordinated Activity of Podoplanin, CD44 and CD9. doi:10.1101/793141.

12. Diz-Muñoz, A. et al. Membrane Tension Acts Through PLD2 and mTORC2 to Limit Actin Network Assembly During Neutrophil Migration. PLOS Biol. 14, (2016).

13. De Belly, H. et al. Membrane Tension Gates ERK-Mediated Regulation of Pluripotent Cell Fate. Cell Stem Cell 28, 273-284.e6 (2021).

14. Compan, V. et al. Cell Volume Regulation Modulates NLRP3 Inflammasome Activation. Immunity 37, 487-500 (2012). 


\section{Movies S1 to S4}

S1A - The paracortical T-cell FRC network, showing 3D localisation of actomyosin structures in homeostasis.

Staining of the conduit (perlecan, magenta), FRC network (PDPN, yellow), F-actin (phalloidin, cyan) and $\mathrm{pMLC}$ (red). PDPN and perlecan surface renders show the location of actomyosin in relation to these structures.

S1B - Laser ablation of homeostatic FRC network.

Movies captured over 25 seconds. Yellow line marks site of cut one second after imaging began.

S2A - Laser ablation of FRC network day 3 post immunisations.

Movies captured over 25 seconds. Yellow line marks site of cut one second after imaging began.

S2B - Laser ablation of FRC network day 5 post immunisations.

Movies captured over 25 seconds. Yellow line marks site of cut one second after imaging began.

S2C - The paracortical T-cell FRC network, showing 3D localisation of actomyosin structures day 3 post immunisations.

Staining of the conduit (perlecan, magenta), FRC network (PDPN, yellow), F-actin (phalloidin, cyan) and PMLC (red). PDPN and perlecan surface renders show the location of actomyosin in relation to these structures.

S2D - The paracortical T-cell FRC network, showing 3D localisation of actomyosin structures day 5 post immunisation.

Staining of the conduit (perlecan, magenta), FRC network (PDPN, yellow), F-actin (phalloidin, cyan) and PMLC (red). PDPN and perlecan surface renders show the location of actomyosin in relation to these structures.

S3A - Optical tweezers generate membrane tethers used to determine membrane tension

S4A - Osmotic swelling of PDPN CTRL fibroblastic reticular cells

PDPN CTRL + CLEC2 (left), PDPN CTRL - CLEC2 (middle), PDPN CTRL ISO control (right). Captured over 60 minutes with one frame every 30 seconds.

S4B - Osmotic swelling of PDPN CTRL fibroblastic reticular cells

B) PDPN T34A + CLEC2 (left), PDPN T34A - CLEC2 (middle), PDPN T34A ISO control (right). Captured over 60 minutes with one frame every 30 seconds. 
bioRxiv preprint doi: https://doi.org/10.1101/2021.05.27.446027; this version posted July 30, 2021. The copyright holder for this preprint (which

was not certified by peer review) is the author/funder, who has granted bioRxiv a license to display the preprint in perpetuity. It is made available under aCC-BY-NC-ND 4.0 International license.

S4C - Osmotic swelling of PDPN CTRL fibroblastic reticular cells

C) CTRL + HYPO (left), PDPN KD + HYPO (middle), CTRL ISO control (right). Captured over 60 minutes with one frame every 30 seconds.

S4D - Osmotic swelling of PDPN CTRL fibroblastic reticular cells

D) $\mathrm{CTRL}+\mathrm{H}_{2} \mathrm{O}$ (left), PDPN KD $+\mathrm{H}_{2} \mathrm{O}$ (right). Captured over 60 minutes with one frame every 30 seconds. 